\title{
The first WIMPy halos
}

\author{
Anne M. Green $\uparrow \S$, Stefan Hofmann£and Dominik J. Schwarz\| \\ $\dagger$ Physics and Astronomy, University of Sheffield, Sheffield S3 7RH, UK \\ $\ddagger$ Astronomy Centre, Department of Physics and Astronomy, University of Sussex, \\ Falmer, Brighton, BN1 9QH, UK \\ $\S$ Physics Department, Stockholm University, 10691 Stockholm, Sweden \\ || Fakultät für Physik, Universität Bielefeld, Postfach 100131, 33501 Bielefeld, \\ Germany \\ E-mail: a.m.green@sheffield.ac.uk, stehof@physto.se, \\ dschwarz@physik.uni-bielefeld.de
}

\begin{abstract}
.
Dark matter direct and indirect detection signals depend crucially on the dark matter distribution. While the formation of large scale structure is independent of the nature of the cold dark matter $(\mathrm{CDM})$, the fate of inhomogeneities on sub-galactic scales, and hence the present day CDM distribution on these scales, depends on the micro-physics of the CDM particles. We study the density contrast of Weakly Interacting Massive Particles (WIMPs) on sub-galactic scales. We calculate the damping of the primordial power spectrum due to collisional damping and freestreaming of WIMPy CDM and show that free-streaming leads to a CDM power spectrum with a sharp cut-off at about $10^{-6} M_{\odot}$. We also calculate the transfer function for the growth of the inhomogeneities in the linear regime, taking into account the suppression in the growth of the CDM density contrast after matter-radiation equality due to baryons and show that our analytic results are in good agreement with numerical calculations. Combining the transfer function with the damping of the primordial fluctuations we produce a WMAP normalized primordial CDM power spectrum, which can serve as an input for high resolution CDM simulations. We find that the smallest inhomogeneities typically have co-moving radius of about $1 \mathrm{pc}$ and enter the non-linear regime at a redshift of $60 \pm 20$. We study the effect of scale dependence of the primordial power spectrum on these numbers and also use the spherical collapse model to make simple estimates of the properties of the first generation of WIMP halos to form. We find that the very first WIMPy halos may have a significant impact on indirect dark matter searches.
\end{abstract}

Keywords: dark matter, cosmological perturbation theory 


\section{Introduction}

Analysis of the anisotropies in the cosmic microwave background (CMB) radiation [1] finds that the relative matter density $\Omega_{\mathrm{m}}=0.29 \pm 0.07$ is significantly larger than the relative baryon density $\Omega_{\mathrm{b}}=0.047 \pm 0.006$. This is consistent with the observed abundances of light elements and primordial nucleosynthesis (see e.g. Reference [2]) and the power spectrum found from galaxy red-shift surveys [3], and indicates that the Universe contains a significant amount of non-baryonic cold dark matter (CDM). The identification of the nature of the CDM particles is a major outstanding issue for cosmology (and would provide reassuring confirmation of the CDM cosmological paradigm).

There are various CDM candidates (for a recent review see 4]), the most well studied of which are Weakly Interacting Massive Particles (WIMPs) and axions. WIMPs are a particularly attractive CDM candidate, since a stable relic from the electroweak scale generically has an interesting present day density, $\Omega_{\text {wimp }} \sim \mathcal{O}(1)$ [5]. There are a large number of ongoing experiments attempting to detect WIMPs directly in the lab [6] or indirectly via their annihilation products (gamma-rays, antiprotons and neutrinos) 7.

The signals expected in dark matter detection experiments depend in most cases on the distribution of the dark matter. Direct detection experiments probe the dark matter distribution on sub-milli-pc scales [8, 9] while indirect detection signals are strongest from the highest density regions of the Milky Way (see e.g. References [8, 10]) and the extra-galactic gamma-ray signal depends on the clumpiness of the WIMP distribution [11]. Reliable predictions for the expected signals therefore require an understanding of the clumpiness of dark matter on small (sub-galactic) scales. The density perturbations on very small scales, and hence the properties of the first generation of structures to form, depend on the microphysics of the CDM and the present day density distribution may retain traces of these first structures.

Two of the present authors showed that the microphysics of WIMPs (specifically collisional damping due to interactions with the radiation component and freestreaming) lead to a fundamental small scale cut-off in the WIMP density perturbation power spectrum 12, 13. Subsequently we presented the small scale WIMP density, velocity and potential perturbations and estimated the properties of the first generation of WIMP halos to form for the case of the WIMP being a bino [14. Meanwhile Berenzinsky et al. [15] studied the survival probability of these first halos analytically. More recently Diemand et al. 16] have carried out high resolution simulations of the first WIMP halos to form and there has been (inconclusive) discussion about whether these halos will be disrupted by interactions with stars [17. Shortly after the first version of the present work became available on arXiv.org, Loeb and Zaldarriaga published a numerical calculation of the cut-off scale [18. Their work is an improvement on the analytic treatment in the present paper, although the estimates for the cut-off mass scales agree up to a factor of order unity (the difference between their $10^{-4} M_{\odot}$ and our 
$10^{-6} M_{\odot}$ stems largely from different assumptions for the kinetic decoupling temperature - $10 \mathrm{MeV}$ in [18] versus $30 \mathrm{MeV}$ for our benchmark model in [14, which is a somewhat more realistic value for the lightest neutralino).

In this paper we present the detailed calculations behind the results presented in our earlier letter 14 generalised to generic WIMPs, including a new more intuitive collisional damping calculation using the Navier-Stokes equation. We also compare our analytic transfer function with numerical calculations and study the effects of a scale dependent primordial power spectrum. In Section 2 we estimate the temperature scales related to chemical and kinetic decoupling for generic WIMPs. In Section 3 we define the fluid perturbation variables and present the equations necessary for our subsequent calculations and solve the evolution of the matter and radiation perturbations during the radiation dominated regime. In Sections 4 and 5 we calculate the damping of the WIMP density contrast due, respectively, to collisional damping (as a result of elastic interactions with other species) and free-streaming. We then (Section 6) calculate the transfer functions, which encode the evolution of the perturbations, by solving the evolution equations around matter-radiation equality and matching these solutions to the sub-horizon limits of the radiation domination solutions found in Section 3, and compare our expressions with the output of the COSMICS package. This allows us to calculate the power spectrum for the WIMP perturbations on sub-galactic scales shortly after matter-radiation equality (Section (7). We consider the case of a scale-invariant primordial power spectrum and discuss the modifications due to tilt and running of the spectral index for single-field inflationary models. Finally in Section 8 we estimate the epoch at which typical-sized inhomogeneities go non-linear for a range of benchmark WIMP models and examine the effects of scale dependence of the primordial power spectrum on our results. To conclude we also estimate the epoch when the very first (rare) inhomogeneities go non-linear and estimate their size after collapse and speculate about their fate and relevance for dark matter searches.

\section{WIMPs}

Weakly interacting massive particles are generic candidates for CDM. The reason is that they are quite natural in extensions of the standard model of particle physics, the most popular example being supersymmetry. In supersymmetry models every standard model particle has a supersymmetric partner and in most models there is a conserved quantum number (R-parity), which makes the lightest supersymmetric particle stable. Supersymmetry models have a large number of free parameters, however in most models the lightest supersymmetric particle is the lightest neutralino (which is a mix of the supersymmetric partners of the photon, the $Z$ and the Higgs bosons) and an excellent CDM candidate (see e.g. Reference [19]). We have focused our attention on the neutralino (in particular a bino-like neutralino) in our previous work 14. Here we wish to dwell on the model-independent aspects of WIMP astrophysics. Our treatment is valid given three assumptions hold true: we assume that there is no WIMP anti- 
WIMP asymmetry in the universe and we assume that WIMPs have been in chemical and thermal equilibrium with the radiation component in the early (hot) Universe (thus Wimpzillas are different from what we call generic WIMPs). The third assumption is that the elastic cross sections are dominated by $Z^{0}$ exchange. This assumption is wrong for bino-like neutralinos, but as our previous studies have shown, the final result is not very sensitive to this detail. For simplicity, we also assume that there is only one CDM component, namely WIMPs.

In the early universe WIMPs can be treated as an ideal Bose or Fermi gas characterised by $g$ internal degrees of freedom, the WIMP mass $m$, chemical potential $\mu$ and temperature $T$. Unless the WIMPs have a net non-zero quantum number (in which case a mechanism is required to generate a WIMP anti-WIMP asymmetry) the chemical potential is negligible and, independent of the spin statistics, for $T \ll m$ and $\mu \ll m$ the distribution function takes the Boltzmann form and the relative relic abundance of WIMPs is given by:

$$
\Omega_{\text {wimp }}=\left.\frac{2}{3}\left(\frac{2^{3}}{\pi}\right)^{1 / 2} \frac{g_{\mathrm{s}} T^{3}}{\left(m_{\mathrm{Pl}} H\right)^{2}}\right|_{0} \frac{g m}{\left.g_{\mathrm{s}}\right|_{\mathrm{cd}}}\left(x_{\mathrm{cd}}\right)^{3 / 2} \exp \left(-x_{\mathrm{cd}}\right) \cosh \left(x_{\mathrm{cd}} \frac{\mu_{\mathrm{cd}}}{m}\right) .
$$

Here $m_{\mathrm{Pl}}$ denotes the Planck mass, $H$ the Hubble rate, $g_{s}$ the number of relativistic degrees of freedom contributing to the total entropy of the radiation fluid and $x \equiv m / T$. The subscripts ' 0 ' and 'cd' refer to the present day and the epoch at which WIMP antiWIMP annihilation ceased and the WIMPs chemically decoupled, respectively. In the following we assume $\mu_{\mathrm{cd}}=0$.

Equation (II) can be solved iteratively for $x_{\mathrm{cd}}$ :

$$
\begin{aligned}
& x_{\mathrm{cd}} \approx x_{\mathrm{cd}}^{(0)}+\frac{3}{2} \ln x_{\mathrm{cd}}^{(0)}, \\
& x_{\mathrm{cd}}^{(0)} \approx 23+\ln \left(\frac{m}{100 \mathrm{GeV}}\right)-\ln \left(\Omega_{\mathrm{wimp}} h^{2}\right)+\ln (g)+\ln \left(\frac{\left.g_{\mathrm{s}}\right|_{0}}{\left.g_{\mathrm{s}}\right|_{\mathrm{cd}}}\right) .
\end{aligned}
$$

After chemical decoupling, $T \lesssim T_{\text {cd }}$ the total number of WIMPs remains constant and they are kept in local thermal equilibrium by elastic scattering processes with relativistic particles: WIMP $+\mathrm{L} \longleftrightarrow$ WIMP $+\mathrm{L}$. As the Universe expands the WIMP density decreases, the elastic scattering rate decreases and the WIMPs kinetically decouple. The characteristic time scale between elastic scatterings is $\tau_{\mathrm{f}} \equiv 1 / \Gamma_{\mathrm{el}}$, where $\Gamma_{\mathrm{el}}$ denotes the elastic scattering rate. The average momentum exchanged per collision is small, of order $T$ [20, 12], and the number of elastic scatterings needed to keep the WIMPs in local thermal equilibrium, $N$, is large: $N \approx m / T \gg 1$. The relaxation timescale, $\tau_{\mathrm{r}}$, which characterizes the time at which the WIMP kinetically decouple, is given by $\tau_{\mathrm{r}} \equiv N \tau_{\mathrm{f}}$ and is significantly larger than the elastic scattering timescale.

The elastic scattering rate is given by

$$
\Gamma_{\mathrm{el}} \equiv \sum_{\mathrm{L} \in \mathrm{SM}}\left\langle v \sigma_{\mathrm{el}}\right\rangle n_{\mathrm{L}}
$$

where $\sigma_{\mathrm{el}}$ is the total cross section for elastic scatterings of WIMPs and relativistic Standard Model fermions, $n_{\mathrm{L}}$ the number density of relativistic particles of species $L$, 
which are assumed to be in local thermal equilibrium and $v \approx 1$ in this case. The thermal average of $\sigma_{\mathrm{el}}$ can be written as $\left\langle\sigma_{\mathrm{el}}\right\rangle=\sigma_{0}^{\mathrm{el}}(T / m)^{1+l}$, where $\sigma_{0}^{\mathrm{el}} \approx\left(G_{\mathrm{F}} m_{\mathrm{W}}^{2}\right)^{2} m^{2} / m_{\mathrm{Z}}^{4}$ sets the scale for the cross section and $l$ parametrises the temperature dependence. Here, $m_{\mathrm{W}}$ denotes the mass of the charged gauge bosons in the electroweak interaction, $m_{\mathrm{Z}}$ is the mass of the neutral gauge boson and $G_{\mathrm{F}}$ is Fermi's coupling constant. In the Standard Model, elastic scattering between a light fermion and a heavy fermion is mediated by $\mathrm{Z}^{0}$ exchange and $l=0$. Other channels may occur however. In supersymmetric extensions of the Standard Model, where the lightest neutralino is a WIMP candidate, sfermion exchange occurs (and if the neutralino is a gaugino, $\mathrm{Z}^{0}$ exchange is suppressed), in which case $l=1$.

Kinetic decoupling of WIMPs happens at a temperature $T_{\mathrm{kd}}$, defined by $\tau_{\mathrm{r}}\left(T_{\mathrm{kd}}\right)=$ $H^{-1}\left(T_{\mathrm{kd}}\right)$. Solving this equation for $x_{\mathrm{kd}} \equiv m / T_{\mathrm{kd}}$, we find

$$
\begin{aligned}
x_{\mathrm{kd}} & \approx\left[\frac{\zeta(3)}{\pi^{2}}\left(\frac{\left.90 g_{\epsilon}\right|_{\mathrm{kd}}}{8 \pi^{3}}\right)^{1 / 2} m_{\mathrm{Pl}} m \sigma_{0}^{\mathrm{el}}(m)\right]^{\frac{1}{3+l}}, \\
& \approx\left[\left.7 \cdot 10^{13} g_{\epsilon}^{1 / 2}\right|_{\mathrm{kd}}\left(\frac{m}{100 \mathrm{GeV}}\right)^{3}\right]^{\frac{1}{3+l}},
\end{aligned}
$$

where $g_{\epsilon}^{1 / 2}$ is the number of degrees of freedom contributing to the energy density. For $l=0$ the kinetic decoupling temperature does not depend on the WIMP mass and is given by $\left.T_{\mathrm{kd}} \approx 2.4 g_{\epsilon}^{-1 / 6}\right|_{\mathrm{kd}} \mathrm{MeV}$. For $l=1,\left.T_{\mathrm{kd}} \approx 34.2 g_{\epsilon}^{-1 / 8}\right|_{\mathrm{kd}}(m / 100 \mathrm{GeV})^{1 / 4} \mathrm{MeV}$.

In figure 1 we plot the dependence of the WIMP chemical and kinetic decoupling temperatures on the WIMP mass, for WIMPs with relic densities corresponding to the WMAP measurement of the CDM density (i.e. assuming that the cold dark matter is entirely in the form of WIMPs) $\omega_{\mathrm{cdm}}=\Omega_{\mathrm{cdm}} h^{2}=0.076-0.156$, consistent with the 2-sigma error of WMAP [1].

While chemical decoupling happens at a temperature of around $10 \mathrm{GeV}$, kinetic decoupling is delayed by the large entropy of the hot Universe and takes place at around $10 \mathrm{MeV}$. This is generic for any WIMP that is of cosmological relevance today.

\section{Cosmological perturbations of fluids}

Fluctuations in the energy density of radiation and matter come together with fluctuations of the scalar metric potentials. In the conformal Newtonian (or longitudinal or zero shear) gauge, perturbations of an isotropic and homogeneous, spatially flat metric are characterised by two scalar potentials $\phi$ and $\psi$ which appear in the line element as (e.g. Reference [21])

$$
\mathrm{d} s^{2}=a^{2}(\eta)\left[-(1+2 \phi) \mathrm{d} \eta^{2}+(1-2 \psi) \delta_{i j} \mathrm{~d} x^{i} \mathrm{~d} x^{j}\right],
$$

where $a$ denotes the scale factor and $\eta$ conformal time. We work in this gauge since all sub-horizon quantities can be interpreted in terms of Newtonian physics, in particular the scalar potential $\phi$ is identical to the gravitational potential in the Newtonian limit. This is in contrast to the synchronous gauge where the Newtonian gravitational 


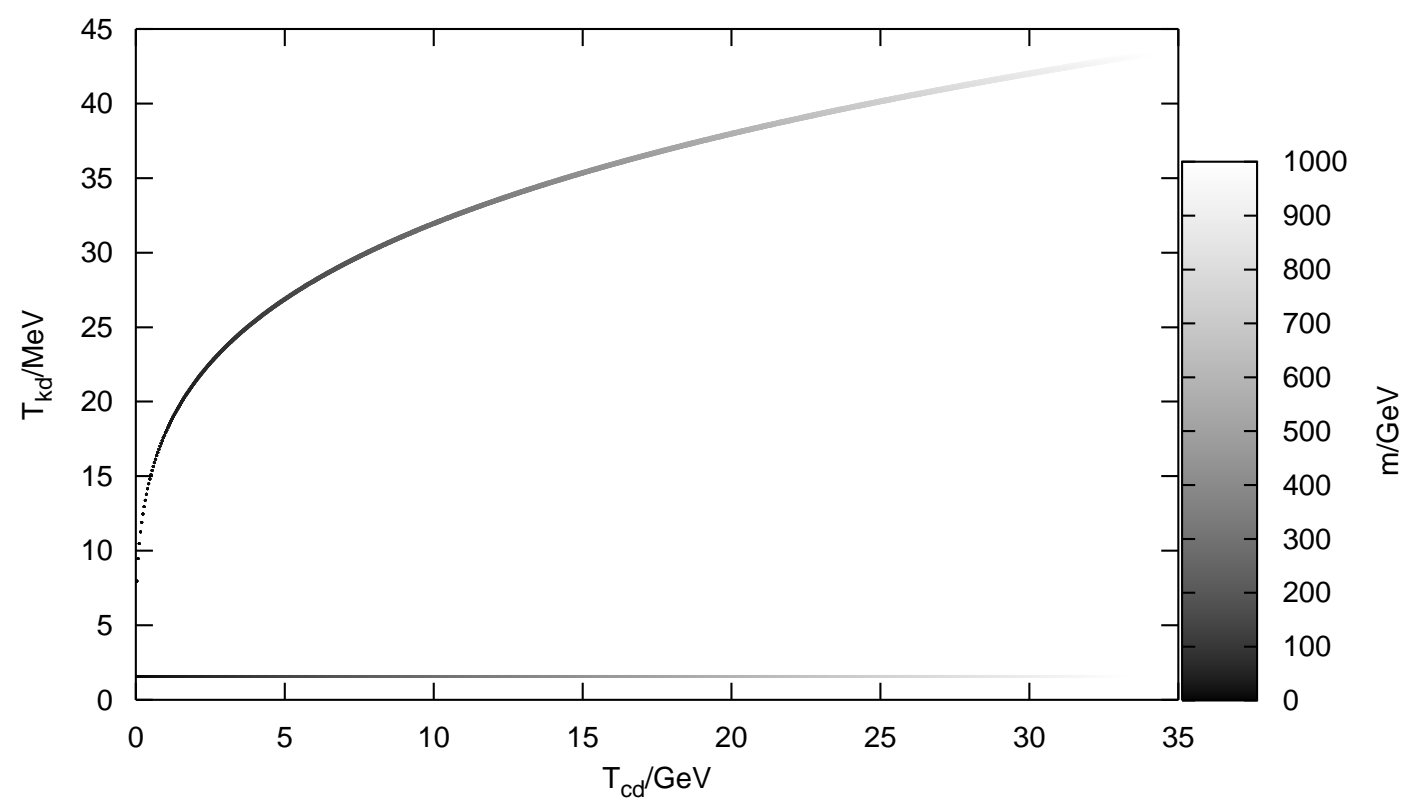

Figure 1. The dependence of the WIMP chemical and kinetic decoupling temperatures, $T_{\mathrm{cd}}$ and $T_{k d}$, on the WIMP mass (indicated by the grey scale) for WIMPs with $\omega_{\text {wimp }}=0.076-0.156$. The upper and lower bands are for $l=1$ (Majorana) and 0 (Dirac) respectively.

potential is gauged to zero. Furthermore, in the conformal Newtonian gauge there is no complicating residual gauge degree of freedom.

For an isotropic fluid with energy density $\epsilon$, pressure $P$ and four-velocity $u^{\mu}$, the components of the energy-momentum tensor are given by

$$
T_{\nu}^{\mu}=(\epsilon+P) u^{\mu} u_{\nu}+P \delta_{\nu}^{\mu} .
$$

Perturbations in the energy density and pressure are denoted by $\delta \epsilon$ and $\delta P$ respectively, and we treat the (small) fluid peculiar velocity $v_{i}$ as a linear velocity perturbation. The peculiar velocity is related to the spatial component of the four-velocity by $u^{i}=v^{i} / a$. The components of the perturbed energy-momentum tensor are given to first order in the perturbations by

$$
\delta T_{0}^{0}=-\delta \epsilon, \quad \delta T_{j}^{0}=\frac{1}{a}(\epsilon+P) v_{j}, \quad \delta T_{j}^{i}=\delta_{j}^{i} \delta P+\Pi_{j}^{i}
$$

Here, $\Pi_{j}^{i} \equiv T_{j}^{i}-\delta_{j}^{i} T_{k}^{k} / 3$ is the traceless anisotropic pressure. Quantum fluctuations during inflation do not seed anisotropic pressure, however once neutrinos decouple from the photon-lepton fluid at about $T \sim 1 \mathrm{MeV}$, they build up anisotropic pressure, which has to be taken into account to obtain the correct normalisation of the power spectra. 
We will include this effect below, but neglect the anisotropic stress of the neutrinos for all other aspects in this paper.

In a spatially flat background it is convenient to consider Fourier modes with wavenumber $\mathbf{k}$. For linear perturbations these modes are decoupled from each other and, due to the isotropy and homogeneity of the background, all modes with the same $k=|\mathbf{k}|$ obey identical mode equations.

We define dimensionless scalars to characterise the fluctuations of each fluid $\Delta_{\mathrm{a}}$

$$
\Delta_{\mathrm{a}} \equiv \frac{\delta \epsilon_{\mathrm{a}}}{(\epsilon+P)_{\mathrm{a}}},
$$

and $v_{\mathrm{a}}$, the modulus of the peculiar velocity,

$$
v_{\mathrm{a}} \equiv-\imath \hat{\mathbf{k}} \cdot \mathbf{v}_{\mathrm{a}},
$$

with the index index "a" denoting the type of fluid (e.g. radiation $P_{\mathrm{r}}=\epsilon_{\mathrm{r}} / 3$ or CDM $\left.P_{\text {cdm }}=0\right)$.

It is also useful to define the total energy density contrast $\Delta$ and peculiar velocity modulus $v$ :

$$
\Delta=\sum_{\mathrm{a}} \frac{(\epsilon+P)_{\mathrm{a}}}{\epsilon+P} \Delta_{\mathrm{a}}, \quad v=\sum_{\mathrm{a}} \frac{(\epsilon+P)_{\mathrm{a}}}{\epsilon+P} v_{\mathrm{a}},
$$

which act as sources of the energy and momentum constraints respectively. For fluids, the pressure can be related to the energy density and the entropy by an equation of state $P_{\mathrm{a}}=P_{\mathrm{a}}\left(\epsilon_{\mathrm{a}}, S_{\mathrm{a}}\right)$. We need only consider the situation of adiabatic and isentropic evolution of each fluid, thus $\delta S_{\mathrm{a}}=0$, and hence

$$
\delta P_{\mathrm{a}}=c_{a}^{2} \delta \epsilon_{\mathrm{a}}, \quad c_{\mathrm{a}}^{2}=\left(\frac{\partial P_{\mathrm{a}}}{\partial \epsilon_{\mathrm{a}}}\right)_{S},
$$

where $c_{\mathrm{a}}$ is the adiabatic sound speed of fluid "a".

Each of the fluids individually (if they are noninteracting and dissipationless) obeys the general relativistic continuity and Euler equations. These equations read (here we have used $c_{\mathrm{a}}^{2}=P_{\mathrm{a}}^{\prime} / \epsilon_{\mathrm{a}}^{\prime}$, which is valid for linear perturbations only):

$$
\begin{aligned}
\Delta_{\mathrm{a}}^{\prime} & =k v_{\mathrm{a}}+3 \psi^{\prime}, \\
v_{\mathrm{a}}^{\prime}+\left(1-3 c_{\mathrm{a}}^{2}\right) \mathcal{H} v_{\mathrm{a}} & =-c_{\mathrm{a}}^{2} k \Delta_{\mathrm{a}}-k \phi+\frac{2}{3} k \pi_{\mathrm{a}},
\end{aligned}
$$

where the primes denote derivatives with respect to conformal time $\eta$ and $\mathcal{H} \equiv a^{\prime} / a$. Anisotropic pressure is generated by $\pi$ which is defined by $\Pi_{j}^{i} /(\epsilon+P)=\left(\delta_{j}^{i} / 3-\hat{k}^{i} \hat{k}_{j}\right) \pi$.

Any number of perfect fluids can be described by one effective imperfect fluid. The effective dimensionless entropy perturbation is

$$
\mathcal{S} \equiv \frac{\delta P-c_{s}^{2} \delta \epsilon}{\epsilon+P}=\sum_{a} c_{\mathrm{a}}^{2} \frac{(\epsilon+P)_{\mathrm{a}}}{\epsilon+P}\left(\Delta_{\mathrm{a}}-\Delta\right)
$$

where the effective adiabatic sound speed is given by

$$
c_{\mathrm{s}}^{2}=\sum_{a} c_{\mathrm{a}}^{2} \frac{(\epsilon+P)_{\mathrm{a}}}{\epsilon+P} \text {. }
$$


For a Universe containing CDM and radiation fluids we find

$$
\mathcal{S}=\frac{y}{4+3 y}\left(\Delta_{\mathrm{r}}-\Delta_{\mathrm{cdm}}\right), \quad c_{\mathrm{s}}^{2}=\frac{1}{3} \frac{1}{1+3 y / 4},
$$

where $y \equiv a / a_{\mathrm{eq}}$ is the scale factor normalised at matter-radiation equality.

We can now define isentropic initial conditions by demanding that $\mathcal{S}=0$ and $\mathcal{S}^{\prime}=0$. The first condition gives $\Delta_{\mathrm{cdm}}=\Delta_{\mathrm{r}}$ and the second, making use of the continuity equation (13), gives $v_{\text {cdm }}=v_{\mathrm{r}}$ additionally. The generalisation to an arbitrary number of fluids is straightforward. Below we restrict our attention to these (isentropic) initial conditions.

In order to close the set of equations we also need the Einstein equations; the background equation

$$
\mathcal{H}^{2}-\mathcal{H}^{\prime}=4 \pi G a^{2}(\epsilon+P),
$$

and the energy and momentum constraints, which have $\Delta$ and $v$ as source terms respectively,

$$
\begin{aligned}
-k^{2} \psi-3 \mathcal{H} \psi^{\prime}-3 \mathcal{H}^{2} \phi & =\left(\mathcal{H}^{2}-\mathcal{H}^{\prime}\right) \Delta, \\
-k\left(\psi^{\prime}+\mathcal{H} \phi\right) & =\left(\mathcal{H}^{2}-\mathcal{H}^{\prime}\right) v .
\end{aligned}
$$

Combining the spatial trace of the Einstein equations and the energy constraint one can write down an equation which has $\mathcal{S}$ as a source (see e.g. Reference [21]), but we do not need it here. The spatial off-diagonal Einstein equation couples the anisotropic pressure to the metric potentials, such that

$$
k^{2}(\psi-\phi)=2\left(\mathcal{H}^{2}-\mathcal{H}^{\prime}\right) \pi .
$$

For isotropic fluids, $\pi=0$ and hence $\psi=\phi$. In general the difference between $\psi$ and $\phi$ can be neglected on subhorizon scales $(k \gg \mathcal{H})$.

To normalise the spectra of cosmological perturbations, we have to connect solutions in the radiation, matter and dark energy dominated epochs with the primordial fluctuations that are produced during the epoch of cosmological inflation. To do that it is most convenient to introduce a quantity that is independent of the choice of the hypersurface of constant time and is constant for modes in the superhorizon regime $(k \ll \mathcal{H})$. Following Bardeen $[22$ we define

$$
\zeta=\Delta / 3-\psi
$$

which is the curvature perturbation on uniform density hypersurfaces or the density contrast on uniform curvature hypersurfaces. We see immediately from the continuity equations (12), that this quantity could be defined for each fluid or for one effective fluid and that it is constant for regular solutions on superhorizon scales (assuming that there are no entropy perturbations) [23. In the following we normalise the power spectrum of $\zeta$ to the value measured by WMAP, where this variable is denoted $-\mathcal{R}$.

During radiation domination $\left(\epsilon_{\mathrm{r}} \gg \epsilon_{\mathrm{cdm}}\right)$, the perturbation equations (12) and (13) can be solved exactly for the cold dark matter and isotropic radiation $(\pi=0 \Rightarrow \phi=\psi)$ 
fluids, on all scales [20. In the conformal Newtonian gauge we find for the dominant growing mode

$$
\begin{aligned}
\phi^{\mathrm{rad}}(\kappa) & =-2 \zeta_{0} \frac{j_{1}(\kappa)}{\kappa}, \\
\Delta_{\mathrm{r}}^{\mathrm{rad}}(\kappa) & =3 \zeta_{0}\left[j_{0}(\kappa)-2 \frac{j_{1}(\kappa)}{\kappa}+\kappa j_{1}(\kappa)\right], \\
v_{\mathrm{r}}^{\mathrm{rad}}(\kappa) & =\sqrt{3} \zeta_{0}\left[\kappa j_{0}(\kappa)-2 j_{1}(\kappa)\right], \\
\Delta_{\mathrm{cdm}}^{\mathrm{rad}}(\kappa) & =6 \zeta_{0}\left[\ln (\kappa)+j_{0}(\kappa)-\frac{j_{1}(\kappa)}{\kappa}-\operatorname{Ci}(\kappa)+\gamma_{\mathrm{E}}-\frac{1}{2}\right], \\
v_{\mathrm{cdm}}^{\mathrm{rad}}(\kappa) & =2 \sqrt{3} \zeta_{0} \frac{1-j_{0}(\kappa)}{\kappa},
\end{aligned}
$$

where $\kappa \equiv k \eta / \sqrt{3}, \zeta_{0}$ is the value of $\zeta$ in the superhorizon limit, $\gamma_{\mathrm{E}}$ Euler's constant, Ci the cosine integral and $j_{n}$ are the spherical Bessel functions, all defined as in [24]. It is easy to check that this is the isentropic mode, since in the superhorizon limit $\Delta_{\mathrm{cdm}} \rightarrow \Delta_{\mathrm{r}}$ and $v_{\mathrm{cdm}} \rightarrow v_{\mathrm{r}}$.

On superhorizon scales $(k \ll \mathcal{H}), \phi \simeq-2 \zeta_{0} / 3, \Delta_{\mathrm{r}} \simeq \zeta_{0}, v_{\mathrm{r}} \simeq(k / \mathcal{H}) \zeta_{0} / 3$. On subhorizon scales $(k \gg \mathcal{H})$, a first order expansion of the exact solutions, (22) and (23), in $\kappa$ gives

$$
\begin{aligned}
& \phi^{\mathrm{rad}}(\kappa) \simeq 2 \zeta_{0} \frac{\cos (\kappa)}{\kappa^{2}}, \\
& \Delta_{\mathrm{r}}^{\mathrm{rad}}(\kappa) \simeq-3 \zeta_{0} \cos (\kappa), \quad v_{\mathrm{r}}^{\mathrm{rad}}(\kappa) \simeq \sqrt{3} \zeta_{0} \sin (\kappa), \\
& \Delta_{\mathrm{cdm}}^{\mathrm{rad}}(\kappa) \simeq 6 \zeta_{0}\left[\ln (\kappa)+\gamma_{\mathrm{E}}-\frac{1}{2}\right], \quad v_{\mathrm{cdm}}^{\mathrm{rad}}(\kappa) \simeq \frac{2 \sqrt{3} \zeta_{0}}{\kappa} .
\end{aligned}
$$

During radiation domination, the radiation density and velocity perturbations on subhorizon scales oscillate with constant amplitude, thereby generating a Newtonian gravitational potential that decays like $1 / \kappa^{2}$, while the CDM density perturbations grow logarithmically and the velocity perturbations decay with $1 / \kappa$. From (24) it can be seen, that the Newtonian gravitational potential acts like an external field on the evolution of CDM density perturbations in this regime.

In the next section we discuss the viscous coupling of WIMPs to radiation and its effect on the evolution of WIMP density perturbations during radiation domination. While the viscous coupling has important consequences for the spectrum of WIMP perturbations, the evolution of the radiation fluid is unaffected.

\section{Kinetic decoupling}

Elastic scattering processes around $T_{\mathrm{kd}}$ lead to viscous coupling of the WIMP and radiation fluids, which results in collisional damping of WIMP perturbations, as shown by two of us in 12. The WIMP perturbations disperse due to bulk and shear viscosity. The strength of these damping mechanisms is described in terms of local transport coefficients $\zeta_{\text {vis }}$ and $\eta_{\text {vis }}$ for bulk and shear viscosity. Close to local thermal equilibrium, 
$\zeta_{\text {vis }} \approx 5 / 3 n T \tau_{\text {relax }}$ and $\eta_{\text {vis }} \approx n T \tau_{\text {relax }}$. The WIMP sound speed in that regime is given by $\sqrt{(5 / 3)(T / m)}$. The WIMP perturbations that survive the viscous coupling prior to kinetic decoupling are then the initial conditions for the free streaming regime.

We now calculate the spectrum of WIMP density perturbations on the surface of kinetic decoupling by solving the linearised Navier-Stokes equations. For a more general and complete derivation, see [12. Dissipation is a subhorizon phenomenon. The linearised Navier-Stokes equation for the WIMP fluid on subhorizon scales $(k \gg \mathcal{H})$, is given by

$$
v_{\text {wimp }}^{\prime}+\frac{\zeta_{\text {vis }}+4 / 3 \eta_{\text {vis }}}{\epsilon_{\text {wimp }}} \frac{k^{2}}{a} v_{\text {wimp }}=-c_{\text {wimp }}^{2} k \Delta_{\text {wimp }},
$$

where a gravitational forcing term and energy dissipation due to the large-scale inhomogeneities of the radiation fluid, as well as horizon scale contributions, have been dropped. Similarly, the continuity equation (12) becomes $\Delta_{\text {wimp }}^{\prime}=k v_{\text {wimp. }}$. These equations can be combined to give a second order differential equation for the evolution of $\Delta_{\text {wimp }}$ :

$$
\Delta_{\text {wimp }}^{\prime \prime}+\frac{\zeta_{\text {vis }}+4 / 3 \eta_{\text {vis }}}{\epsilon_{\text {wimp }}} \frac{k^{2}}{a} \Delta_{\text {wimp }}^{\prime}+c_{\text {wimp }}^{2} k^{2} \Delta_{\text {wimp }}=0 .
$$

Note that the final term should not be neglected as its coefficient $\left(c_{\text {wimp }}^{2} \sim T / m\right)$ is of comparable magnitude to that of the dissipative term $\left[(T / m)\left(\tau_{\text {relax }} / t\right)\right]$ for $t \sim \tau_{\text {relax }}$. This is the equation of motion of an oscillator with time dependent friction, due to the bulk and shear viscosity, and hence $\Delta_{\text {wimp }}$ oscillates with complex frequency. The real part of the frequency is proportional to the isentropic sound speed in the WIMP fluid and describes the propagation of perturbations in this fluid, while the imaginary part describes the damping of the perturbations caused by the transfer of energy and momentum, due to the viscous coupling, from the WIMP fluid to the radiation fluid (which acts as a heat bath).

Let us stress that our treatment is restricted to $k \gg \mathcal{H}$. Near the horizon there are extra forcing terms on the right hand side of (27). There are two types of such terms. Firstly, there is the gravitational pull of the oscillating radiation fluid. Secondly, as long as $c_{\text {wimp }} \sim c_{\text {rad }}$ the oscillations of the radiation fluid can drag the CDM fluid along by means of multiple scatterings in a preferred direction. As soon as the relaxation time scale exceeds the oscillation period of a given mode, this mechanism can no longer be active. This coherent effect is not accounted for by our viscosity terms, which reflect the dissipation due to the homogeneous component of the radiation fluid. There are also some extra terms from the Hubble expansion on the left hand side of (27).

The numerical treatment of Loeb and Zaldarriaga [18] includes these terms. Their results confirms that the essential features of the subhorizon damping are captured by our calculation. They also show that the additional terms play an important role in determining the precise position of the maximum of the CDM power spectrum. We agree with their conclusion that an accurate calculation (better than 10\% accuracy) must be based on a numerical computation including all of the terms. 
The WKB solution to (27) for the envelope of the WIMP density oscillations is:

$$
\Delta_{\text {wimp }}\left(k, \eta_{\mathrm{kd}}\right)=\Delta_{\text {wimp }}\left(k, \eta_{\mathrm{i}}\right) \exp \left(-\int_{\eta_{\mathrm{i}}}^{\eta_{\mathrm{kd}}} \frac{\zeta_{\text {vis }}+4 / 3 \eta_{\text {vis }}}{2 \epsilon_{\text {wimp }}} \frac{k^{2}}{a} \mathrm{~d} \eta\right),
$$

where $\Delta_{\text {wimp }}\left(k, \eta_{\mathrm{i}}\right)$ is the initial primordial density perturbation and $\eta_{\mathrm{kd}}$ is the conformal time at kinetic decoupling. The damping term can be written as

$$
D_{\mathrm{d}}(k) \equiv \frac{\Delta_{\text {wimp }}\left(k, \eta_{\mathrm{kd}}\right)}{\Delta_{\text {wimp }}\left(k, \eta_{\mathrm{i}}\right)}=\exp \left[-\left(\frac{k}{k_{\mathrm{d}}}\right)^{2}\right]
$$

where $k_{\mathrm{d}}$ is given by

$$
\begin{aligned}
k_{\mathrm{d}} & =\left(\int_{\eta_{\mathrm{i}}}^{\eta_{\mathrm{kd}}} \frac{\zeta_{\mathrm{vis}}+4 / 3 \eta_{\mathrm{vis}}}{2 \epsilon_{\mathrm{wimp}} a} \mathrm{~d} \eta\right)^{-1 / 2}, \\
& =\left(\frac{3}{2} \int_{\eta_{\mathrm{i}}}^{\eta_{\mathrm{kd}}} \frac{T}{m} \frac{\tau_{\text {relax }}}{a} \mathrm{~d} \eta\right)^{-1 / 2}, \\
& \approx 1.8\left(\frac{m}{T_{\mathrm{kd}}}\right)^{1 / 2} \frac{a_{\mathrm{kd}}}{a_{0}} H_{\mathrm{kd}}, \\
& \approx \frac{3.76 \times 10^{7}}{\mathrm{Mpc}}\left(\frac{m}{100 \mathrm{GeV}}\right)^{1 / 2}\left(\frac{T_{\mathrm{kd}}}{30 \mathrm{MeV}}\right)^{1 / 2} .
\end{aligned}
$$

This scale corresponds to a length scale $\sim 10^{-2} / H$ at kinetic decoupling. The total WIMP mass contained in a sphere with radius $\pi / k_{\mathrm{d}}$ is $M_{\mathrm{d}} \sim 10^{-10} M_{\odot}$. Here we assumed a WIMP mass typical for the lightest supersymmetric particle and a typical kinetic decoupling temperature for a weakly interacting particle of such a mass [12, 14].

Once the WIMPs have kinetically decoupled, they can be described as a separate fluid. On scales larger than the free streaming length, we can approximate it as a pressureless fluid. Since kinetic decoupling happens well before the end of radiation domination the gravitational evolution of the WIMP density perturbations on arbitrary scales during radiation domination $\left(\epsilon_{\mathrm{m}} \ll \epsilon_{\mathrm{r}}\right)$ is given by (23) .

\section{Free streaming}

After kinetic decoupling the evolution of the WIMPs at the smallest scales is described by the distribution function $f(\mathbf{x}, q \mathbf{n}, \eta)$, where $q$ and $\mathbf{n}$ are the norm and direction of the comoving 3-momentum, i.e. $q_{\mathrm{ph}}=q / a$. The distribution function is governed by the collisonless Boltzmann equation, which reads in a flat Friedmann model (see e.g. [25])

$$
\left(\frac{\partial}{\partial \eta}+\frac{\mathrm{d} x^{i}}{\mathrm{~d} \eta} \frac{\partial}{\partial x^{i}}+\frac{\mathrm{d} q}{\mathrm{~d} \eta} \frac{\partial}{\partial q}+\frac{\mathrm{d} n_{j}}{\mathrm{~d} \eta} \frac{\partial}{\partial n_{j}}\right) f(\mathbf{x}, q \mathbf{n}, \eta)=0 .
$$

In local thermal equilibrium we have $f(\mathbf{x}, q \mathbf{n}, \eta)=f_{0}(q, \eta)$, which in the case of non-relativistic particles becomes the Maxwell-Boltzmann distribution function (with $g$ internal degrees of freedom),

$$
f_{\mathrm{MB}}(q)=\frac{g}{(2 \pi)^{3}} \exp \left(\frac{\mu-m}{T_{\text {wimp }}}\right) \exp \left[-\frac{(q / a)^{2} / 2 m}{T_{\text {wimp }}}\right] \text {. }
$$


Here, $T_{\text {wimp }}$ denotes the temperature and $\mu$ the chemical potential of the WIMPs. Both depend on time, but $f_{\mathrm{MB}}(q)$ does not.

In the next step we have to investigate deviations of the distribution function of the WIMPs in order to describe an inhomogeneous universe. We will do so by considering small perturbations in the Boltzmann equation away from the local thermal equilibrium solution. Close to local thermal equilibrium we can write

$$
f(\mathbf{x}, q \mathbf{n}, \eta)=f_{0}(q, \eta)+\delta f(\mathbf{x}, q \mathbf{n}, \eta) .
$$

The last term in (31) does not contribute at first order in the perturbed quantities. This is because $\mathrm{d} n_{j} / \mathrm{d} \eta$ and $\partial f / \partial n_{j}$ are individually first order terms; in the absence of metric perturbations free-streaming particles do not change direction and the zero-th order distribution function $f_{0}(q, \eta)$ is independent of direction $\mathbf{n}$. Let us now turn to the third term in (31). From the geodesic equation it follows that $\mathrm{d} q / \mathrm{d} \eta$ is proportional to derivatives of metric perturbations. Free streaming only occurs on scales well below the horizon (due to the small velocities of WIMPs) and on subhorizon scales the metric perturbations $\phi$ and $\psi$ are negligible - consequently the third term in (31) can also be neglected. The collisionless Boltzmann equation for subhorizon scales can then be rewritten in terms of the spatial Fourier transform of $\delta f(\mathbf{x}, q \mathbf{n}, \eta)$, which we denote by $\delta f(\mathbf{k}, q \mathbf{n}, \eta)$ :

$$
\left(\frac{\partial}{\partial \eta}+i \frac{q / a}{m} \mathbf{k} \cdot \mathbf{n}\right) \delta f(\mathbf{k}, q \mathbf{n}, \eta)=0,
$$

Note that the second term in this equation depends on the direction $\mathbf{n}$ of the comoving momentum only via its angle with respect to the wavenumber $\mathbf{k}$.

Before solving equation (34) the initial perturbations have to be specified. At kinetic decoupling we can assume that $T=T_{\text {wimp }}$ and we drop the index "wimp" in the following. The WIMP phase space distribution around kinetic decoupling is close to a Maxwell-Boltzmann distribution (32). The WIMP density perturbations (with $\mathcal{H} \ll k \ll k_{\mathrm{d}}$ ) which are present on the surface of kinetic decoupling need to be taken into account. To do so we consider small fluctuations $\delta T$ and $\delta \mu$ of the thermodynamic variables $T$ and $\mu$ in (32) and expand the resulting distribution up to first order:

$$
\frac{\delta f}{f_{0}}\left(k, q, \eta_{\mathrm{kd}}\right)=\delta\left(\frac{\mu}{T}\right)+\left(\frac{m}{T}+\frac{(q / a)^{2} / 2 m}{T}\right) \frac{\delta T}{T}+\mathcal{O}\left(\delta^{2}\right) .
$$

As we are interested only in effects of first order in the perturbed quantities, the adiabatic equations of motion can be used to establish the relationships between temperature and chemical potential fluctuations as well as WIMP perturbations on the surface of kinetic decoupling. From the conservation of entropy per WIMP we find $\delta n / n \approx(3 / 2) \delta T / T$ and thus $\delta T / T \approx 2 \Delta_{\text {wimp }}\left(k, \eta_{\mathrm{kd}}\right) / 3$. Inserting this in the Gibbs-Duhem relation $\delta(\mu / T)=\delta P /(n T)-(\epsilon+P) /(n T)(\delta T / T)$ and using the previous relation, we find $\delta(\mu / T) \approx-(2 m / 3 T) \Delta_{\text {wimp }}\left(k, \eta_{\mathrm{kd}}\right)$, so that the deviation from local thermal equilibrium at kinetic decoupling is given by

$$
\left(\frac{\delta f}{f_{0}}\right)\left(k, q, \eta_{\mathrm{kd}}\right)=\frac{q_{\mathrm{kd}}^{2} / 2 m}{3 T_{\mathrm{kd}} / 2} \Delta_{\text {wimp }}\left(k, \eta_{\mathrm{kd}}\right)+\mathcal{O}\left(\delta^{2}\right) .
$$


This has an obvious interpretation, the fractional perturbation of the distribution function is proportional to the ratio of the kinetic energy of an individual particle with comoving velocity $\mathrm{q} / \mathrm{m}$ and the thermal energy $3 T / 2$ times the density contrast.

The solution to the free streaming equation (34) is then

$$
\left(\frac{\delta f}{f_{0}}\right)(\mathbf{k}, q \mathbf{n}, \eta)=\left(\frac{\delta f}{f_{0}}\right)\left(k, q, \eta_{\mathrm{kd}}\right) \exp [-\mathrm{i} l(q, \eta) \mathbf{k} \cdot \mathbf{n}],
$$

where $l=l(q, \eta)$ is the comoving distance a WIMP can travel freely in the background space-time during the time interval $\eta-\eta_{\mathrm{kd}}$ :

$$
l(q, \eta)=\int_{\eta_{\mathrm{kd}}}^{\eta} \mathrm{d} \eta^{\prime} \frac{q / m}{a\left(\eta^{\prime}\right)} .
$$

We can evaluate that this integral for the matter-radiation universe, in which we can write the scale factor as

$$
a(\eta)=a_{\mathrm{eq}}\left[1 / 4\left(\frac{\mathcal{H}_{\mathrm{eq}} \eta}{\sqrt{2}}\right)^{2}+\frac{\mathcal{H}_{\mathrm{eq}} \eta}{\sqrt{2}}\right] .
$$

Using the previously introduced notation $y=a / a_{\text {eq }}$ some algebra finally yields,

$$
l(q, \eta)=\frac{q_{\mathrm{kd}}}{m} y_{\mathrm{kd}} \ln \left[\frac{y_{\mathrm{kd}}}{y} \frac{y+2(1-\sqrt{1+y})}{y_{\mathrm{kd}}+2\left(1-\sqrt{1+y_{\mathrm{kd}}}\right)}\right] \frac{\sqrt{2}}{\mathcal{H}_{\mathrm{eq}}},
$$

where $q_{\mathrm{kd}} \equiv q / a_{\mathrm{kd}}$ is the modulus of the physical momentum at kinetic decoupling. As $y \ll 1$, the physical distance of travel of free streaming particles approaches $\left[\left(q_{\mathrm{kd}} / m\right) y_{\mathrm{kd}}\right] \sqrt{2} \ln \left(4 / y_{\mathrm{kd}}\right)\left[a / \mathcal{H}_{\mathrm{eq}}\right]$, the first square bracket is the physical velocity at kinetic decoupling and the scale is set by the physical size of the Hubble horizon at equality (the other term in square brackets).

The free streaming of the WIMPs generates a further (collisionless) damping mechanism for WIMP density perturbations. After averaging over the Maxwellian distribution of velocities, we can estimate the damping scale by replacing the physical velocity of an individual WIMP by the mean velocity (thermal velocity) of WIMPs at kinetic decoupling. Thus the comoving length scale of free streaming

$$
l_{\mathrm{fs}} \sim \bar{v}_{\mathrm{kd}} a_{\mathrm{kd}} \int_{\eta_{\mathrm{kd}}}^{\eta} \frac{\mathrm{d} \eta^{\prime}}{a\left(\eta^{\prime}\right)}
$$

with $\bar{v}_{\mathrm{kd}}=\sqrt{3 g T / m}$. In order to characterise the spectrum of perturbations which survive collisionless damping we introduce the comoving free streaming scale $k_{\mathrm{fs}} \propto 1 / l_{\mathrm{fs}}$. More precisely we define

$$
k_{\mathrm{fs}}(\eta) \equiv \frac{\sqrt{2}}{\sqrt{T_{\mathrm{kd}} / m}\left[l\left(q_{\mathrm{kd}}, \eta\right) /\left(q_{\mathrm{kd}} / m\right)\right]},
$$

where the numerical prefactor is chosen for later convenience. With the help of (40) we find that the comoving $k_{\mathrm{fs}}$ becomes approximately constant as $a / a_{\mathrm{eq}} \gg 1$ and is given by

$$
k_{\mathrm{fs}} \approx\left(\frac{m}{T_{\mathrm{kd}}}\right)^{1 / 2} \frac{a_{\mathrm{eq}} / a_{\mathrm{kd}}}{\ln \left(4 a_{\mathrm{eq}} / a_{\mathrm{kd}}\right)} \frac{a_{\mathrm{eq}}}{a_{0}} H_{\mathrm{eq}}
$$




$$
\approx \frac{1.70 \times 10^{6}}{\mathrm{Mpc}} \frac{(m / 100 \mathrm{GeV})^{1 / 2}\left(T_{\mathrm{kd}} / 30 \mathrm{MeV}\right)^{1 / 2}}{1+\ln \left(T_{\mathrm{kd}} / 30 \mathrm{MeV}\right) / 19.2} .
$$

This expressions depends on $\omega_{\mathrm{m}} \equiv \Omega_{\mathrm{m}} h^{2}$ only through the logarithm, we therefore set it equal to the WMAP best fit value, $\omega_{\mathrm{m}}=0.14$ [1]. The corresponding length scale at matter-radiation equality is $\sim 10^{-8} / H$ and the total WIMP mass contained in a sphere of radius $\pi / k_{\mathrm{fs}}$ is $M_{\mathrm{fs}} \sim 10^{-6} M_{\odot}$.

The WIMP density contrast is related to the distribution function by:

$$
\Delta_{\text {wimp }}(\mathbf{k}, \eta)=\frac{\int_{0}^{\infty} \mathrm{d} q q^{2} \int \mathrm{d} \Omega \delta f(\mathbf{k}, q \mathbf{n}, \eta)}{4 \pi \int_{0}^{\infty} \mathrm{d} q q^{2} f_{0}(q, \eta)},
$$

with $\mathrm{d} q q^{2} \mathrm{~d} \Omega$ denoting the volume measure in spherical coordinates in $\mathbf{q}$-space. The denominator in (44) is the comoving mass density of CDM and the numerator is the CDM mass fluctuation due to the deviation from local thermal equilibrium. Using (36) and (37) in (44), we find after integration over $\Omega$

$$
\begin{aligned}
\Delta_{\text {wimp }}(\mathbf{k}, \eta)= & \left(\frac{2}{\pi}\right)^{1 / 2} \Delta_{\text {wimp }}\left(\mathbf{k}, \eta_{\mathrm{kd}}\right)\left(m T_{\mathrm{kd}}\right)^{-3 / 2} \int_{0}^{\infty} \mathrm{d} q_{\mathrm{kd}} q_{\mathrm{kd}}^{2} \frac{q_{\mathrm{kd}}^{2}}{3 m T_{\mathrm{kd}}} \\
& \times \exp \left[-\frac{q_{\mathrm{kd}}^{2}}{2 m T}\right] \frac{\sin \left(l\left(q_{\mathrm{kd}}, \eta\right) k\right)}{l\left(q_{\mathrm{kd}}, \eta\right) k} .
\end{aligned}
$$

The remaining integration can be found as expression 3.952(5) in Reference [26].

As a result of the integration we find the suppression of the WIMP density contrast due to free streaming to be

$$
D_{\mathrm{fs}}(k, \eta) \equiv \frac{\Delta_{\text {wimp }}(\mathbf{k}, \eta)}{\Delta_{\text {wimp }}\left(\mathbf{k}, \eta_{\mathrm{kd}}\right)}=\left[1-\frac{2}{3}\left(\frac{k}{k_{\mathrm{fs}}}\right)^{2}\right] \exp \left[-\left(\frac{k}{k_{\mathrm{fs}}}\right)^{2}\right] .
$$

We see that the free streaming of WIMPs results in exponential damping of the WIMP density contrast, similar to that produced by collisional damping (justifying the terminology 'collisionless damping' for the effects of free streaming). There is one difference, however; the ratio of the WIMP kinetic energy to the thermal averaged kinetic energy in (36) leads to a polynomial pre-factor. This expression is valid for $k / k_{\mathrm{fs}}<1$, as terms of order $\left(k / k_{\mathrm{fs}}\right)^{4}$ have been neglected in the polynomial.

The net damping is the product of the collisional (due to viscous coupling to the radiation fluid) and collisionless (due to free streaming) damping terms [(29) and (46) respectively], $D(k)=D_{\mathrm{d}}(k) D_{\mathrm{fs}}(k)$ :

$$
D(k) \equiv \frac{\Delta_{\text {wimp }}(k, \eta)}{\Delta_{\text {wimp }}\left(k, \eta_{\mathrm{i}}\right)}=\left[1-\frac{2}{3}\left(\frac{k}{k_{\mathrm{fs}}}\right)^{2}\right] \exp \left[-\left(\frac{k}{k_{\mathrm{fs}}}\right)^{2}-\left(\frac{k}{k_{\mathrm{d}}}\right)^{2}\right] .
$$

Since $k_{\mathrm{fs}} \ll k_{\mathrm{d}}$, the cut-off in the power spectrum is determined by the free streaming scale $k_{\mathrm{fs}}$.

In figure 2 we plot the variation of the characteristic damping and free streaming comoving wave numbers with WIMP mass, for WIMPs with relic densities corresponding to the WMAP measurement of the CDM density (i.e. assuming that the cold dark matter is entirely in the form of WIMPs) $\omega_{\mathrm{cdm}}=\Omega_{\mathrm{cdm}} h^{2}=0.076-0.156$, consistent 


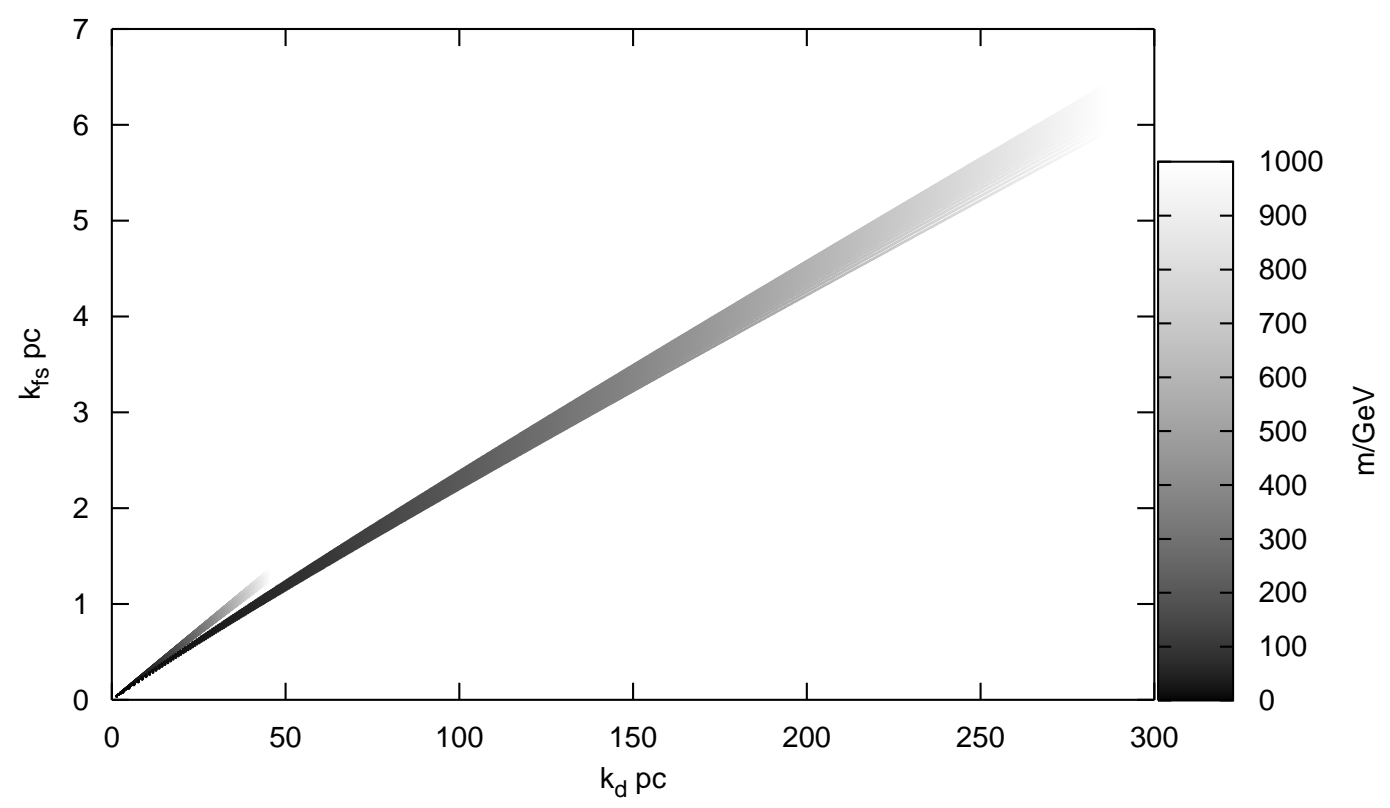

Figure 2. The variation of the characteristic damping and free streaming comoving wavenumbers, $k_{\mathrm{d}}$ and $k_{f s}$, with WIMP mass (indicated by grey scale). The lower and upper bands are for $\mathrm{l}=1$ (Majorana) and 0 (Dirac) respectively.

with the 2-sigma error of WMAP [1]. For concrete calculations we will concentrate on four benchmark models which span the range of most plausible WIMP properties. The details of these benchmark models, including the values of $k_{\mathrm{d}}$ and $k_{\mathrm{fs}}$, are tabulated in Table 1. Models B and C are very close to the bino-like neutralino cases of our previous work [14. Models A and D show that there is more spread of the predicted damping scale if the strong assumption of a bino-like WIMP is dropped.

In order to compare our analytic results with the numerical result of reference [18, we express the cut-off scales in terms of matter mass enclosed in a sphere of radius $R=\pi / k_{\mathrm{fs}}, M_{\text {cut }}(R) \approx 4.9 \times 10^{-6} M_{\odot}(1 / k \mathrm{pc})^{3}$. For a decoupling temperature of 25 $\mathrm{MeV}$, we obtain from [18] a mass of $6.4 \times 10^{-6} M_{\odot}$, which is to be contrasted with $1.5 \times 10^{-6} M_{\odot}$ from our calculation. So, the difference between our analytic result and the numerical result including effects near the horizon scale at decoupling is a factor of a few, instead of two orders of magnitude as claimed in [18].

The approximate equality of the kinetic decoupling and free streaming scales (to within an order of magnitude) can be understood by comparing the corresponding physical length scales at equality (after equality the comoving free streaming scale only grows logrithmically). The free streaming length (41) is given by the product of the 
Table 1. Benchmark WIMP models.

\begin{tabular}{|c|c|c|c|c|c|c|}
\hline Ref. & $l$ & $m(\mathrm{GeV})$ & $T_{\mathrm{cd}}(\mathrm{GeV})$ & $T_{\mathrm{kd}}(\mathrm{Mev})$ & $k_{\mathrm{d}}\left(\mathrm{pc}^{-1}\right)$ & $k_{\mathrm{fs}}\left(\mathrm{pc}^{-1}\right)$ \\
\hline A & 0 & 100 & 3.6 & 1.6 & 14 & 0.42 \\
B & 1 & 50 & 1.9 & 21 & 39 & 0.94 \\
C & 1 & 100 & 3.7 & 25 & 61 & 1.5 \\
D & 1 & 500 & 17 & 37 & 180 & 4.0 \\
\hline
\end{tabular}

WIMP velocity at equality, the logarithmic growth factor and the Hubble radius at equality:

$$
l_{\mathrm{fs}} \sim \bar{v}_{\mathrm{kd}} \frac{a_{\mathrm{kd}}}{a_{\mathrm{eq}}} \ln \frac{a_{\mathrm{eq}}}{a_{\mathrm{kd}}} R_{\mathrm{eq}} .
$$

From (30), the collisional damping length at kinetic decoupling is given by the square root of the product of the viscosity terms with the time of kinetic decoupling, divided by the WIMP mass density. The viscosity terms are proportional to the product of the WIMP mass density, the WIMP sound speed $c_{\text {wimp }}$ squared and the relaxation time. At kinetic decoupling the relaxation time is by definition of order the physical Hubble radius. The collisional damping length at equality is thus

$$
l_{\mathrm{d}} \sim c_{\mathrm{wimp}} R_{\mathrm{kd}} \frac{a_{\mathrm{eq}}}{a_{\mathrm{kd}}} \sim c_{\mathrm{wimp}} \frac{a_{\mathrm{kd}}}{a_{\mathrm{eq}}} R_{\mathrm{eq}},
$$

where we have used $H \propto 1 / a^{2}$ during radiation domination and $c_{\text {wimp }} \sim \sqrt{T_{\mathrm{kd}} / m}$. At kinetic decoupling the sound speed and the average WIMP velocity are approximately equal and therefore $l_{\mathrm{fs}} \approx l_{\mathrm{d}} \ln \left(a_{\mathrm{eq}} / a_{\mathrm{kd}}\right)$, i.e. the length scales are roughly equal at equality.

This can also be seen from the Reynolds number of the CDM fluid which is given by $\operatorname{Re} \equiv 2 R_{H} c_{\text {wimp }} \rho / \eta \sim\left(R_{H} / \tau\right) c_{\text {wimp }} m / T$. At kinetic decoupling we have Re $\sim \sqrt{m / T} \sim 1 / c_{\text {wimp }}$. Thus the sound speed of the CDM (equivalent to the mean particle velocity in the non-relativistic case) at kinetic decoupling plays a fundamental role in both processes. Kinetic effects dominate over friction once Re is large. Thus for $T_{\mathrm{kd}} \sim 10 \mathrm{MeV}$ and $m=O(100 \mathrm{GeV})$ we find Re $\sim 100$. We note that by fluid engineering standards, this is still a pretty small Reynolds number. From this consideration we can also see that kinetic decoupling is a very fast process, as $\operatorname{Re} \sim T^{7 / 2}$ (for the case $l=1$ in Eq. 4).

\section{Evolution of CDM perturbations}

Now we turn to the gravitational growth of CDM perturbations. So far we have calculated the damping of the WIMP density perturbations due to collisional and collisionless damping (47), and the gravitational evolution of CDM perturbations for $\epsilon_{\mathrm{r}} \gg \epsilon_{\mathrm{cdm}}$, (23). What remains is to find a solution for the gravitational evolution of CDM perturbations during the matter and dark energy dominated epochs. In the 
following we find analytic approximations to the radiation-matter and matter-dark energy transitions, which finally can be smoothly joined with (23) to provide the correct normalisation to CMB measurements.

Since we are interested in the subgalactic scales only, we can restrict our attention to the subhorizon modes when $\epsilon_{\mathrm{cdm}}$ becomes comparable to $\epsilon_{\mathrm{r}}$. We include neutrinos in the radiation component in order to allow an analytic treatment, i.e. their anisotropic stress is neglected. This leads to errors of around $10 \%$ [27]. We also neglect the baryon inhomogeneities. At early times the baryons are tightly coupled to the radiation fluid, and photon diffusion damping rapidly erases small-scale perturbations in the baryon fluid at $z \sim 10^{6}$ to $10^{5}$. On small scales the tight coupling breaks down prior to recombination, and the baryon perturbations grow, however $\Delta_{\mathrm{b}} \ll \Delta_{\text {cdm }}$ still [28, 29]. Post decoupling on scales $k>k_{\mathrm{b}} \sim 10^{3} \mathrm{Mpc}^{-1}$ the residual electrons allow transfer of energy between the photon and baryon fluids so that thermal pressure prevents the baryon perturbations from growing, until $z_{\mathrm{b}} \sim 150$ [28, 30]. As we are interested in CDM perturbations on small scales at early times, we can neglect the perturbations in the baryon fluid.

For scales which enter the horizon sufficiently long before matter-radiation equality the logarithmic growth of CDM perturbations provides a situation in which, after some time, $\epsilon_{\mathrm{m}} \Delta_{\mathrm{m}} \gg \epsilon_{\mathrm{r}} \Delta_{\mathrm{r}}$ (i.e. only the CDM perturbations are important) even during radiation domination [31. We also have $\epsilon_{\mathrm{m}} \Delta_{\mathrm{m}} \gg \epsilon_{\mathrm{de}} \Delta_{\mathrm{de}}$, where the index de stands for dark energy. In the following we will assume a cosmological constant, for which $\Delta_{\text {de }} \equiv 0$. Thus we only need to keep $\Delta_{\mathrm{m}}$ in $\Delta$ on the rhs in the Poisson equation (18).

With these two approximations (subhorizon scales and cdm fluctuations dominating as the source in the Poisson equation) we can simplify the equations (12) and (13) to

$$
\Delta_{\mathrm{cdm}}^{\prime}=k v_{\mathrm{cdm}}, \quad v_{\mathrm{cdm}}^{\prime}+\mathcal{H} v_{\mathrm{cdm}}=-k \phi,
$$

and the Poisson equation reads

$$
-k^{2} \phi=4 \pi G a^{2} \epsilon_{\mathrm{cdm}} \Delta_{\mathrm{cdm}} .
$$

It is now most convenient to combine these equations to a single one and to use the scale factor instead of conformal time:

$$
a^{2} \frac{\mathrm{d}^{2} \Delta_{\mathrm{cdm}}}{\mathrm{d} a^{2}}+\frac{3}{2}\left(1-\frac{P}{\epsilon}\right) a \frac{\mathrm{d} \Delta_{\mathrm{cdm}}}{\mathrm{d} a}-\frac{3}{2} \frac{\epsilon_{\mathrm{cdm}}}{\epsilon} \Delta_{\mathrm{cdm}}=0 .
$$

\subsection{Radiation-matter transition}

For the radiation-matter transition (at which point the dark energy, or cosmological constant, is negligible) (152) simplifies further [32]:

$$
y(1+y) \frac{\mathrm{d}^{2}}{\mathrm{~d} y^{2}} \Delta_{\mathrm{cdm}}+\left(1+\frac{3}{2} y\right) \frac{\mathrm{d}}{\mathrm{d} y} \Delta_{\mathrm{cdm}}-\frac{3}{2}\left(1-f_{\mathrm{b}}\right) \Delta_{\mathrm{cdm}}=0,
$$

where $y=a / a_{\mathrm{eq}}=\epsilon_{\mathrm{m}} / \epsilon_{\mathrm{r}}$ and $f_{\mathrm{b}}=\omega_{\mathrm{b}} / \omega_{\mathrm{m}}$ is the baryon fraction, with best fit value from WMAP $f_{b}=0.17$ [1]. The exact solution to this equation is a combination of Legendre functions of the first and second kind:

$$
\Delta_{\mathrm{cdm}}(k, y)=B_{1}(k) P_{\nu}(\sqrt{1+y})+B_{2}(k) Q_{\nu}(\sqrt{1+y}),
$$


with index $\nu\left(f_{b}\right)=\left(\sqrt{25-24 f_{b}}-1\right) / 2$.

We now determine the constants $B_{1,2}(k)$ by matching the $y \ll 1$ expansion of equation (154) to the subhorizon limit of the radiation domination solution (25). We find

$$
\begin{aligned}
& B_{1}(k)=6 \zeta_{0}\left[\ln \left(\frac{k}{k_{\mathrm{eq}}}\right)+b\right], \\
& B_{2}(k)=-12 \zeta_{0},
\end{aligned}
$$

where $k_{\text {eq }} \equiv \mathcal{H}_{\text {eq }}$ and

$$
b\left(f_{\mathrm{b}}\right)=\frac{1}{2} \ln \left(\frac{2^{5}}{3}\right)-\gamma_{\mathrm{E}}-\frac{1}{2}-\frac{2}{\nu}-\frac{2 \Gamma^{\prime}[\nu]}{\Gamma[\nu]},
$$

where $\Gamma^{\prime}[\nu]$ is the derivative of $\Gamma(\nu)$ with respect to $\nu$.

Finally expanding (54) for $y \gg 1$ we find that during matter domination, for scales that enter the horizon significantly before matter-radiation equality and $z<z_{\mathrm{b}}$

$$
\Delta_{\mathrm{cdm}}(y)=6 \zeta_{0} c(\nu) y^{\nu / 2}\left[\ln \left(\frac{k}{k_{\mathrm{eq}}}\right)+b\right]
$$

where

$$
c(\nu)=\frac{\Gamma[1+2 \nu]}{2^{\nu} \Gamma^{2}[1+\nu]},
$$

e.g. $\nu\left(f_{\mathrm{b}}\right)=1.79(2), c\left[\nu\left(f_{\mathrm{b}}\right)\right]=1.36(3 / 2)$ and $b\left(f_{\mathrm{b}}\right)=-1.56(-1.74)$ for $f_{\mathrm{b}}=0.17(0)$. Note that before $z_{\mathrm{b}}$, CDM density perturbations grow as $\Delta_{\text {cdm }} \propto a^{\nu / 2}$. Later the baryons follow the CDM and the matter fluctuations grow as $a$. For the peculiar velocity and the Newtonian gravitational potential we obtain

$$
\begin{aligned}
& v_{\mathrm{cdm}}(y)=\frac{k_{\mathrm{eq}}}{k} \sqrt{\frac{y}{2}} \frac{\mathrm{d}}{\mathrm{d} y} \Delta_{\mathrm{cdm}}(y), \\
& \phi(y)=-\frac{3}{4}\left(\frac{k_{\mathrm{eq}}}{k}\right)^{2}\left(1-f_{\mathrm{b}}\right) \frac{\Delta_{\mathrm{cdm}}(y)}{y} .
\end{aligned}
$$

In the following, we omit the subscript 'cdm'.

For redshifts $z_{\mathrm{eq}}>z>z_{\mathrm{b}}$ (between matter-radiation equality and the epoch at which small-scale baryon perturbations start growing) we find the transfer function for the CDM density perturbations for modes which satisfy $k>k_{\mathrm{b}}$

$$
T_{\Delta}(k, z)=(6 c)^{2}\left[\ln \frac{k}{k_{\mathrm{eq}}}+b\right]^{2}\left(\frac{1+z_{\mathrm{eq}}}{1+z}\right)^{\nu},
$$

and the transfer function for the Newtonian gravitational potential on these scales is given by

$$
T_{\phi}(k, z)=\left[\frac{27\left(1-f_{\mathrm{b}}\right) c}{4}\right]^{2}\left[\ln \frac{k}{k_{\mathrm{eq}}}+b\right]^{2}\left(\frac{k_{\mathrm{eq}}}{k}\right)^{4}\left(\frac{1+z_{\mathrm{eq}}}{1+z}\right)^{\nu-2} .
$$

The transfer function for the velocity depends on the initial time and is therefore not a very useful quantity. 


\subsection{Normalisation including anisotropic stress from neutrinos on superhorizon scales}

Above we have made the assumption that $\phi=\psi$ as we neglect the anisotropic stress of neutrinos, i.e. $\pi_{\nu}=0$. For the normalisation this leads to unacceptably large errors. We therefore take the superhorizon anisotropic stress of neutrinos into account in the normalisation.

Let us define the radiation fraction in neutrinos

$$
\alpha \equiv \frac{\epsilon_{\nu}}{\epsilon_{\mathrm{r}}}=0.405,
$$

after $e^{+} e^{-}$annihilation and for three massless neutrinos. The regular solution for neutrinos at superhorizon scales during the radiation dominant epoch gives 33, 34.

$$
\psi_{0}=\left(1+\frac{2 \alpha}{5}\right) \phi_{0}, \quad \Delta_{\mathrm{r} 0}=\Delta_{\mathrm{cdm} 0}=-\frac{3}{2} \phi_{0},
$$

and thus

$$
\zeta_{0}=-\frac{3}{2}\left(1+\frac{4 \alpha}{15}\right) \phi_{0} .
$$

The conservation of $\zeta$ on superhorizon scales then implies that, for $z<z_{\text {eq }}$ but before the start of dark energy domination, the normalisation of CDM fluctuations on superhorizon scales is given as (now we can again assume $\phi=\psi$ since the neutrino anisotropic stress is now suppressed by $\left.\epsilon_{\nu} / \epsilon_{\mathrm{m}}\right)$

$$
\Delta_{\mathrm{cdm}}=-\frac{9}{5}\left(1+\frac{4 \alpha}{15}\right) \phi_{0} .
$$

When normalising to $\zeta_{0}$, which is provided by the WMAP measurement (encoded as the variable $A$ ), we do not have to take special care as the WMAP value includes the effect of neutrinos on superhorizon scales. However, when comparing our analytic result with the results of the COSMICS code below, we have to include this correction, as the initial condition in the code is $\phi_{0}=-1$.

\subsection{Accuracy of approximations}

In Figure 3 we plot the CDM density contrast at $z=300$ and 100 as a function of comoving wavenumber for the WMAP best fit total matter and baryon densities, $\omega_{\mathrm{cdm}}=0.116, \omega_{\mathrm{b}}=0.024\left(f_{\mathrm{b}}=0.171\right.$ and $\left.b(0.171)=-1.562\right)$, and for zero baryon density, $\omega_{\mathrm{cdm}}=0.14, \omega_{\mathrm{b}}=0.00$, with $h=0.72$, using our analytic expressions and also using the "lingercon" Boltzmann solver from the COSMICS package (which includes massless neutrinos) [35. To allow direct comparison with the output of COSMICS we take initial conditions (during radiation domination) here such that $\Delta_{\text {cdm }}=3 / 2$ (i.e. $\left.\phi_{0}=-1\right)$. The rapid oscillations of $\Delta_{\mathrm{r}}$ on sub-horizon scales means that it is not possible to accurately numerically evolve the coupled perturbation equations for the sub-galactic scales $\left(k \gg 10^{4} k_{\text {eq }}\right)$ that we are interested in. We see that our analytic expression accurately reproduces the shape of $\Delta$ for $k>10^{2} k_{\text {eq }}$ and the normalisation is accurate to $\sim 10 \%$. The accuracy initially increases as $z$ becomes smaller, which can 


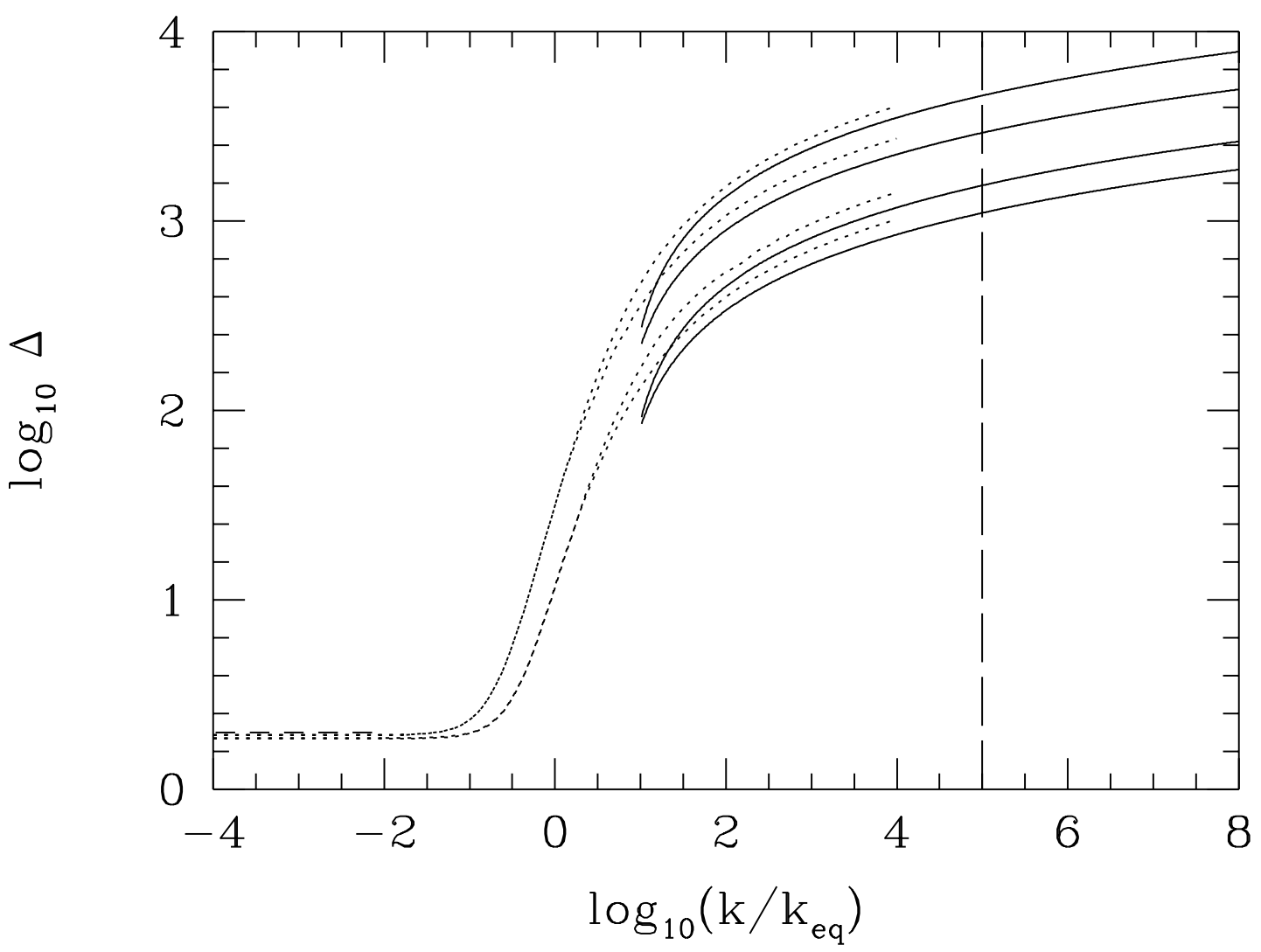

Figure 3. The CDM density contrast $\Delta$ at $z=300$ (lower lines) and $z=100$ (upper lines) for $\omega_{\mathrm{cdm}}=0.116, \omega_{\mathrm{b}}=0.024$ and $\omega_{\mathrm{cdm}}=0.14, \omega_{\mathrm{b}}=0.00$ (bottom and top line of each pair respectively), using our analytic expression (58) (solid line) and also the COSMICS package (dotted line). The analytic superhorizon normalisation (66) is shown as a dashed line for $k<10^{-2} k_{\text {eq }}$. The vertical dashed line denotes $k_{b}$. Our approximation applies best at $k>k_{\mathrm{b}}$ but, as the comparison shows, it is also pretty good for smaller wavenumbers.

be easily understood from the fact that we assumed that $z \ll z_{\text {eq }}$. Our calculation then becomes less accurate for $z<z_{\mathrm{b}}$.

We also see that baryons have a significant effect on the growth of the density contrast; at $z=300 \Delta$ is roughly $40 \%$ smaller in a Universe with the WMAP best fit energy densities $\left(\omega_{\mathrm{cdm}}=0.116, \omega_{\mathrm{b}}=0.024\right)$ than in a Universe with the same total matter density, but no baryons $\left(\omega_{\mathrm{cdm}}=0.14, \omega_{\mathrm{b}}=0.00\right)$.

We conclude that our analytical accuracy is good enough in the light of the present uncertainties in the cosmological parameters and primordial power spectrum.

\subsection{Matter-dark energy transition}

Let us stress before going into any details here, that for the subgalactic scales we are interested in, the following calculation is not directly relevant, since those modes become non-linear long before the onset of dark energy domination. However, we need to take 
the suppression of perturbation growth into account on larger scales when we make contact with the measurement of $\sigma_{8}$ below (section 8).

In $\Lambda$ CDM cosmologies at late times the cosmological constant, or dark energy, dominates the Universe, leading to accelerated expansion and the suppression of the growth of density perturbations. For this situation equation (52) can be simplified neglecting the presence of radiation. We define the scale factor relative to that at the epoch at which the matter and cosmological constant densities are equal: $u=a / a_{\text {eq2 }}$ and $a_{\mathrm{eq} 2}=a_{0}\left(\Omega_{\mathrm{m}} / \Omega_{\Lambda}\right)^{1 / 3}$ and thus $u\left(a_{0}\right)=\left(\Omega_{\Lambda} / \Omega_{\mathrm{m}}\right)^{1 / 3}$. The evolution of the matter density contrast is now governed by

$$
u^{2} \frac{\mathrm{d}^{2}}{\mathrm{~d} u^{2}} \Delta_{\mathrm{m}}+\frac{3}{2}\left(\frac{1+2 u^{3}}{1+u^{3}}\right) u \frac{\mathrm{d}}{\mathrm{d} u} \Delta_{\mathrm{m}}-\frac{3}{2}\left(\frac{1}{1+u^{3}}\right) \Delta_{\mathrm{m}}=0,
$$

which can be obtained from (52) using the change of variables $\Delta_{\mathrm{m}}=u w$ and $u^{3}=-z$. The function $w(z)$ obeys a degenerate hypergeometric differential equation. Its general solution is [see 37, sec. 2.2.2 case 2, eqs. 2.9(1) and 2.9(18) with $a=1, b=1 / 3, c=$ $11 / 6]$

$$
\Delta_{\mathrm{m}}=C_{1}(k) u_{2} F_{1}\left(1, \frac{1}{3} ; \frac{11}{6} ;-u^{3}\right)+C_{2}(k) \sqrt{1+\frac{1}{u^{3}}} .
$$

The first solution is regular for small $u$, the second one is singular. We have to join the regular solution to the growing mode (58). In the limit $u \rightarrow 0$ we have ${ }_{2} F_{1}\left(1,1 / 3 ; 11 / 6 ;-u^{3}\right) \rightarrow 1$ and thus $C_{1}(k)=(3 / 2) B_{1}(k)\left(1+z_{\text {eq }}\right)\left(\Omega_{\Lambda} / \Omega_{\mathrm{m}}\right)^{1 / 3}$ and $C_{2}(k)=0$.

We can now put everything together to obtain the linear matter density contrast today

$$
\Delta_{\mathrm{m}}(k)=9 \zeta_{0}\left(1+z_{\mathrm{eq}}\right)\left(\frac{\Omega_{\Lambda}}{\Omega_{\mathrm{m}}}\right)^{\frac{2}{3}}\left[\ln \left(\frac{k}{k_{\mathrm{eq}}}\right)+b\right]{ }_{2} F_{1}\left(1, \frac{1}{3} ; \frac{11}{6} ;-\frac{\Omega_{\Lambda}}{\Omega_{\mathrm{m}}}\right) .
$$

An excellent analytic approximation to evaluate the hypergeometric function for $\Omega_{\Lambda} / \Omega_{\mathrm{m}}>1$ is given by the asymptotic expansion (see [24])

$$
\begin{aligned}
u_{2} F_{1}\left(1, \frac{1}{3} ; \frac{11}{6} ;-u^{3}\right) & \rightarrow \frac{2 \Gamma\left(\frac{2}{3}\right) \Gamma\left(\frac{11}{6}\right)}{\sqrt{\pi}}\left(1+\frac{1}{2 u^{3}}\right)-\frac{5}{4 u^{2}}+\mathcal{O}\left(\frac{1}{u^{5}}\right) \\
& \approx 1.437-\frac{1.25}{u^{2}}+\frac{0.719}{u^{3}}+\mathcal{O}\left(\frac{1}{u^{5}}\right),
\end{aligned}
$$

which has an accuracy better than $1 \%$ for $\Omega_{\mathrm{m}}<0.4$.

The growth of structure comes to an end asymptotically; in a pure CDM model the density contrast grows as $u$, however in a $\Lambda$ CDM universe it only grows by a factor 1.437 after matter-dark energy equality. An equivalent solution has been obtained by Eisenstein 38 in terms of elliptic functions, however he expanded the elliptical functions for small $u$, which would be more appropriate for $\Omega_{\Lambda} / \Omega_{\mathrm{m}}<1$. One could also write the above hypergeometric function (or elliptical functions of Eisenstein) in terms of associated Legendre functions, but it seems to us that nothing is gained by doing so. 


\section{Linear power spectrum}

In this section we present the linear dimensionless power spectra (defined as $\mathcal{P}_{X}(k, z)=$ $\left.\left(k^{3} / 2 \pi^{2}\right)\left\langle|X(k, z)|^{2}\right\rangle\right)$ normalized to the WMAP measurements ([1, 39]).

\subsection{Scale invariant spectrum}

For simplicity we start with a scale-invariant primordial power spectrum and assume that gravitational waves have a negligible contribution to the CMB anisotropies. We find for $k>k_{\mathrm{b}}$ and $z_{\text {eq }} \gg z \gg z_{\mathrm{b}}$

$$
\begin{aligned}
& \frac{\mathcal{P}_{\Delta}(k, z)}{10^{-7} A}=1.06 c^{2}\left[\ln \frac{k}{k_{\mathrm{eq}}}+b\right]^{2} D^{2}(k)\left(\frac{1+z_{\mathrm{eq}}}{1+z}\right)^{\nu}, \\
& \frac{\mathcal{P}_{v}(k, z)}{10^{-7} A}=0.13 c^{2} \nu^{2}\left[\ln \frac{k}{k_{\mathrm{eq}}}+b\right]^{2}\left(\frac{k_{\mathrm{eq}}}{k}\right)^{2} D^{2}(k)\left(\frac{1+z_{\mathrm{eq}}}{1+z}\right)^{\nu-1}, \\
& \frac{\mathcal{P}_{\phi}(k, z)}{10^{-7} A}=0.60 c^{2}\left(1-f_{\mathrm{b}}\right)^{2}\left[\ln \frac{k}{k_{\mathrm{eq}}}+b\right]^{2}\left(\frac{k_{\mathrm{eq}}}{k}\right)^{4} D^{2}(k)\left(\frac{1+z_{\mathrm{eq}}}{1+z}\right)^{\nu-2}
\end{aligned}
$$

where $A=0.9 \pm 0.1$ at the pivot scale $k_{0}=0.05 / \mathrm{Mpc}$ according to Reference [1]. Note that from the WMAP data the spectral index $n=0.99 \pm 0.04$ is consistent with the scale-invariant Harrison-Zel'dovich spectrum $n=1$. The scale of equality is $k_{\mathrm{eq}}=(0.01 / \mathrm{Mpc})\left(\omega_{\mathrm{m}} / 0.14\right)$ and $1+z_{\mathrm{eq}}=3371\left(\omega_{\mathrm{m}} / 0.14\right)$.

Figure 4 shows the power spectrum for the WIMP density contrast at a redshift of 300 , close to the end of the linear regime of structure formation, with and without the effects of collisional damping and free-streaming for the four benchmark WIMP models introduced in Section 5. In accordance with the WMAP best fit values we take $\omega_{\text {cdm }}=0.116$ and $f_{\mathrm{b}}=0.17$, and we assume a scale invariant primordial power spectrum (i.e. $n=1$ ). It can be observed that the cut-off of the power spectrum is indeed very sharp (with a maximum close to the cut-off). As discussed in section 5 the characteristic free-streaming wave-number is smallest for model A (Dirac WIMP with $\mathrm{l}=0$ ) and increases with increasing mass for the Majorana WIMPs (models B-D). This is clearly reflected in the position of the cut-off in the power spectrum.

\subsection{Scale dependent spectrum}

On the scales probed by the $\mathrm{CMB}\left[\mathcal{O}(0.01-0.1) \mathrm{Mpc}^{-1}\right]$ the primordial power spectrum is close to scale invariant [1]. The free-streaming scale $k_{\mathrm{fs}} \sim 10^{6} \mathrm{Mpc}^{-1}$ is seven orders of magnitude smaller and hence even a very small scale dependence of the power spectrum could significantly change the amplitude of the power spectrum close to the cut-off, and hence the red-shift at which the first WIMP halos form.

To assess the effects of possible scale dependence of the primordial power spectrum we consider three benchmark inflation models which span the range of possible power spectra for simple inflation models: a $V=m^{2} \phi^{2}$ chaotic inflation model, power law 


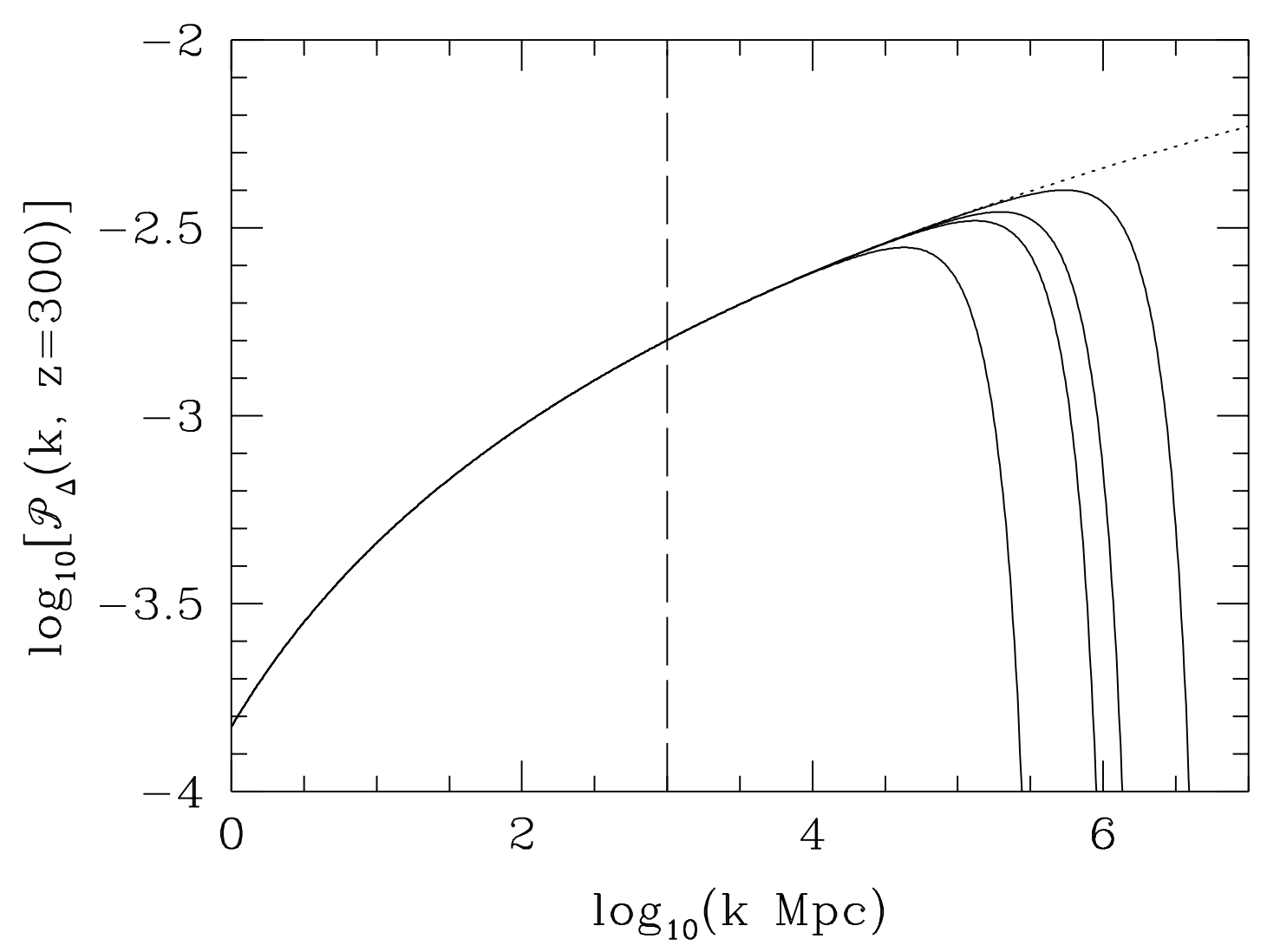

Figure 4. The dimensionless power spectrum of the WIMP density contrast at $z=300$ for our four benchmark WIMP models assuming a scale-invariant primordial power spectrum (full lines, from left to right models A, B, C and D). Without the effects of collisional damping and free streaming, the power spectra would be given by the dotted line. The vertical dashed line denotes $k_{b}$, the wavenumber below which baryons follow CDM. Our approximations are optimised for $k>k_{\mathrm{b}}$.

inflation and a hybrid inflation model. These models span an interesting region of inflationary parameter space, for a more detailed discussion see [40].

There are also uncertainties in the parameterization of the power spectrum. The most commonly used parameterization is

$$
\mathcal{P}(k)=\mathcal{P}\left(k_{0}\right)\left(\frac{k}{k_{0}}\right)^{n\left(k_{0}\right)-1+\frac{1}{2} \alpha\left(k_{0}\right) \ln \left(k / k_{0}\right)},
$$

where $\alpha(k)=\mathrm{d} n / \mathrm{d} k$. An arguably more appropriate parameterization over a wide-range of scales is [41, 42]

$$
\frac{\mathcal{P}(k)}{\mathcal{P}\left(k_{0}\right)}=a_{0}+a_{1} \ln \left(\frac{k}{k_{0}}\right)+\frac{a_{2}}{2} \ln ^{2}\left(\frac{k}{k_{0}}\right) .
$$

A small difference between the two parameterizations can be used as an indicator, that the slow-roll approximation is justified for the model at hand 42.

The spectral index, $n$, its running $\alpha$ and the alternative expansion co-efficients $a_{n}$ depend on the inflationary potential and are most conveniently expressed in terms 
of the horizon flow parameters [41] which are defined as: $\epsilon_{0} \equiv H\left(N_{i}\right) / H(N)$, where $N \equiv \ln \left(a / a_{i}\right)$ is the number of e-foldings of inflation since some initial time $t_{i}$, and

$$
\epsilon_{n+1} \equiv \frac{\mathrm{d} \ln \left|\epsilon_{n}\right|}{\mathrm{d} N} \quad n \geq 1
$$

The horizon flow parameters are related to the traditional slow roll parameters, $\epsilon=\left(m_{\mathrm{pl}}^{2} / 16 \pi\right)\left(V^{\prime} / V\right)^{2}, \eta=\left(m_{\mathrm{pl}}^{2} / 8 \pi\right)\left(V^{\prime \prime} / V\right)$ and $\xi^{2}=\left(m_{\mathrm{pl}}^{2} / 8 \pi\right)^{2}\left(V^{\prime} V^{\prime \prime \prime} / V^{2}\right)$ where $' \equiv \mathrm{d} / \mathrm{d} \phi$, as $\epsilon_{1}=\epsilon, \epsilon_{2}=2 \epsilon-2 \eta$ and $\epsilon_{2} \epsilon_{3}=4 \epsilon^{2}-6 \epsilon \eta+2 \xi^{2}$.

To first order $n-1=-2 \epsilon_{1}-\epsilon_{2}$ (see e.g. Ref. 43] for the higher order terms) and $\alpha=-2 \epsilon_{1} \epsilon_{2}-\epsilon_{2} \epsilon_{3}$ [41]. $a_{0}, a_{1}$ and $a_{2}$ are given by equations (26) - (28) of Ref. 42.

7.2.1. $m^{2} \phi^{2}$ chaotic inflation In this model $\epsilon_{1}=\epsilon_{2} / 2=\epsilon_{3} / 2=1 /(2 N+1)$ (see e.g. Ref. 44]) and we take $N=55$ [45]. This gives, including the higher order terms in the expression for $n, n-1=-0.03611$ and $\alpha=-6.49 \times 10^{-4}$.

7.2.2. Power law inflation In power law inflation the scale factor grows as $a \propto t^{p}$ (with $p>1$ ) and $\epsilon_{1}=1 / p$ with all other horizon flow parameters equal to zero. We pick $p=55.4$ so that the spectral index is the same as for the $m^{2} \phi^{2}$ chaotic inflation model $(n-1=-0.03611)$. In this case there is no running of the power spectrum $(\alpha=0)$, i.e. the spectral index is constant.

\subsubsection{Hybrid inflation Our benchmark hybrid inflation model has potential}

$$
V=V_{0}\left[1+\frac{1}{2}\left(\frac{\phi}{m_{\mathrm{Pl}}}\right)^{2}\right],
$$

and we assume that the first, false vacuum term, in the potential dominates so that $\epsilon_{1} \ll \epsilon_{2}$, with $\epsilon_{2}$ constant. We take $\epsilon_{2}=-0.014$, the $2-\sigma$ lower limit from WMAP and $2 \mathrm{dF}$ found in Ref. [44] giving the largest increase in the density contrast with increasing $k$, so that $n-1=0.03550$ and $\alpha=0$.

The primordial power spectra of these three models are plotted in Figure 5 for both parameterizations of the power spectra (equations (174) and (175)). We see that the amplitude of the primordial power spectra on the free-streaming scale $\sim 10^{6} \mathrm{Mpc}^{-1}$ varies by a factor of $\sim 2.5$ (equivalently the amplitude of the fluctuations varies by $\sim \sqrt{2.5} \sim 1.6$ ). We also observe that the two parameterisations do not give rise to a significant difference, thus we will stick to the more traditional power-law shape in the following.

In figure 6 we plot the processed power spectra for these primordial power spectra and also a scale invariant primordial power spectrum for WIMP benchmark C, with cosmological parameters fixed to the WMAP best fit values as before. The variation in the amplitude of the primordial power spectra for $k \sim 10^{6} \mathrm{Mpc}^{-1}$ translates directly into a variation in the peak amplitude of the processed power spectra. 


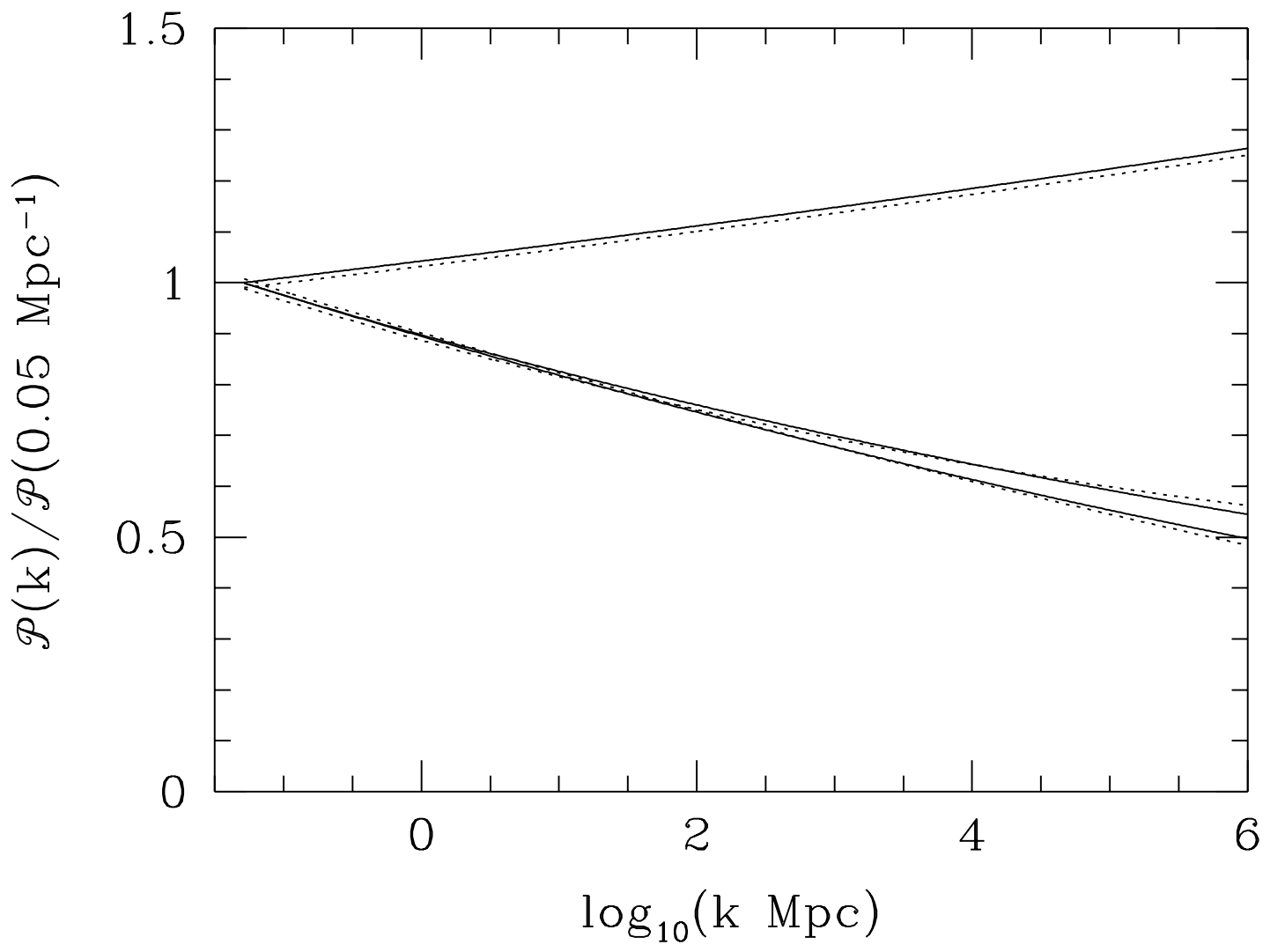

Figure 5. The primordial power spectra of the three benchmark inflation models discussed in the text (from top to bottom: hybrid, power law and $m^{2} \phi^{2}$ chaotic inflation) for the standard power law parameterisation of the power spectrum (equation (74), solid line) and for the expansion in $\ln \left(k / k_{0}\right)$ (equation (75), dotted line).

\section{The first structures}

The collisional damping and free streaming of WIMPs lead to a cut-off in the WIMP power spectrum, which sets the typical scale for the first halos in the hierarchical picture of structure formation. We estimate the redshift at which typical fluctuations on comoving scale $R$ go nonlinear via

$$
\sigma\left(R, z_{\mathrm{nl}}\right)=1,
$$

where $\sigma(R, z)$ is the mass variance defined by

$$
\sigma^{2}(R, z)=\int_{0}^{\infty} W^{2}(k R) \mathcal{P}_{\Delta}(k, z) \frac{\mathrm{d} k}{k},
$$

where $W(k R)$ is the Fourier transform of the window function divided by its volume. In accordance with the usual procedure, we take the window function to be a top hat. We normalize $\sigma(R, z)$ to $\sigma_{8} \equiv \sigma(8 / h \mathrm{Mpc}, 0)=0.9 \pm 0.1$ [1], taking into account the suppression of the growth of $\Delta$ at late times due to the cosmological constant (see section 6.4), as our analytic calculation of the transfer function breaks down for modes close 


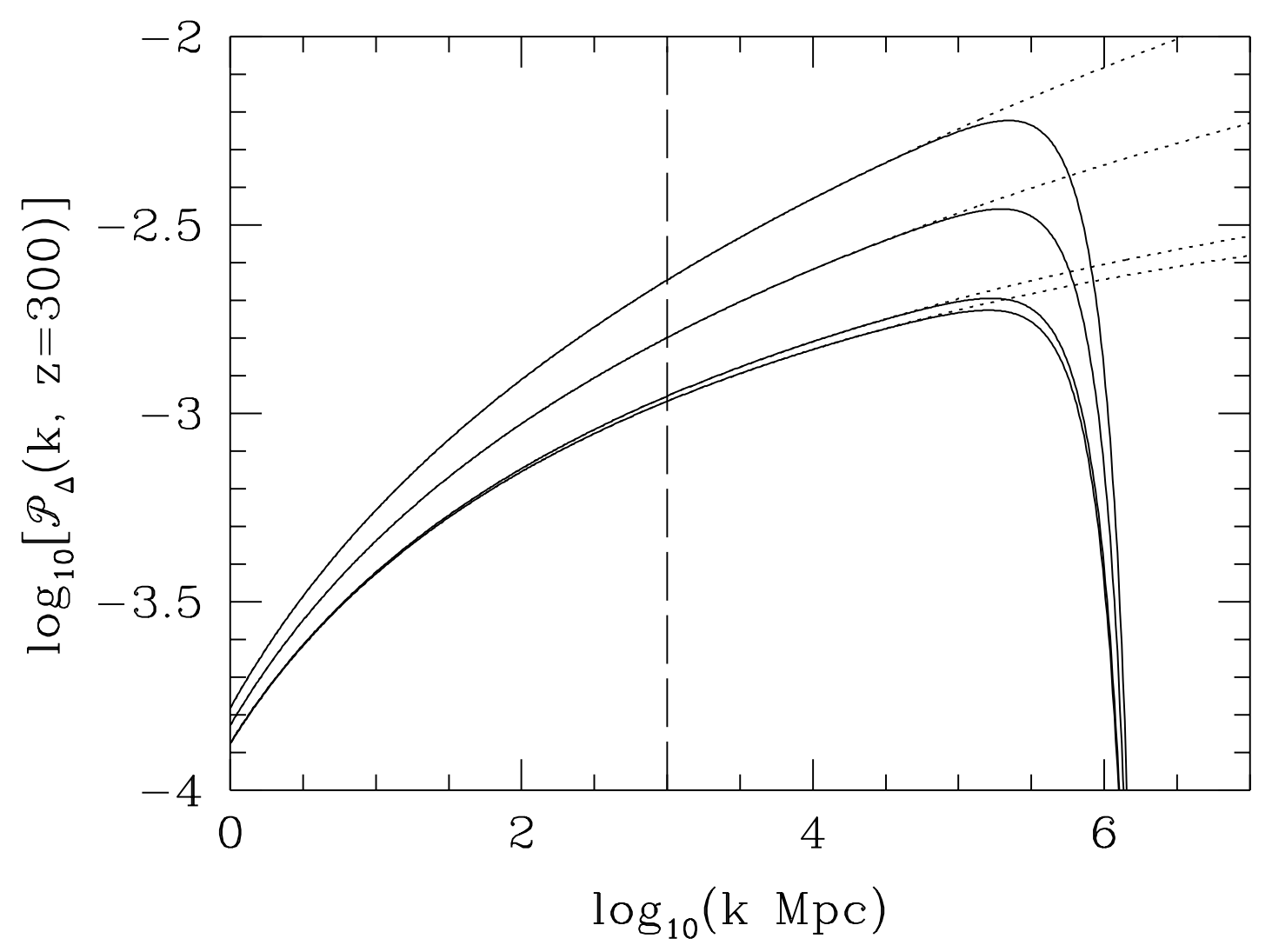

Figure 6. The processed power spectra for the three benchmark inflation models and also a scale invariant primordial power spectrum for WIMP benchmark C (from top to bottom: hybrid inflation, scale invariant primordial spectrum, power law and $m^{2} \phi^{2}$ chaotic inflation). As before the dotted lines are the power spectra without the effects of collisional damping and free streaming and the vertical dashed line denotes $k_{b}$.

to $k_{\text {eq }}$. For the purpose of estimating $z_{\mathrm{nl}}$ we ignore the effects of baryons (see section 6.1 and 6.31) and assume a $\Lambda$ plus matter universe with $\Omega_{\mathrm{m}}$ and $\Omega_{\Lambda}$ as determined by WMAP [1]. Specifically, we take $\Omega_{\mathrm{m}} / \Omega_{\Lambda}=0.370$.

Figures 17 and 8 show $z_{\mathrm{nl}}$, as defined by equation (17), as a function of the scale $R$, for the benchmark WIMPs and primordial power spectra respectively. In each case the cut-off in the processed power spectrum at $k \sim 10^{6} \mathrm{Mpc}^{-1}$ leads to a plateau with $z_{\mathrm{nl}}=z_{\mathrm{nl}}^{\max }$ at $R<R_{\min }=\mathcal{O}(1) \mathrm{pc}$.

We can now give a more precise picture of the onset of the hierarchical structure formation process; non-linear structure formation starts at a redshift $z_{\mathrm{nl}}^{\max }$. For the benchmark WIMP models we see that the order of magnitude variation in $k_{\mathrm{fs}}$ leads to a similar variation in $R_{\min }$ and also (because of the dependence of the amplitude of the peak of the power spectrum on the cut-off scale, see figure 4) a range of values $z_{\mathrm{nl}}^{\max } \approx 50$ to 65. For the scale-dependent primordial power spectra the factor of 1.6 (see section 7.2) variation in the amplitude of the fluctuations on scales $k \sim 10^{6} \mathrm{Mpc}^{-1}$ translates directly into a comparable range of values for $z_{\mathrm{nl}}^{\max }$. Thus, for plausible WIMP properties and for 


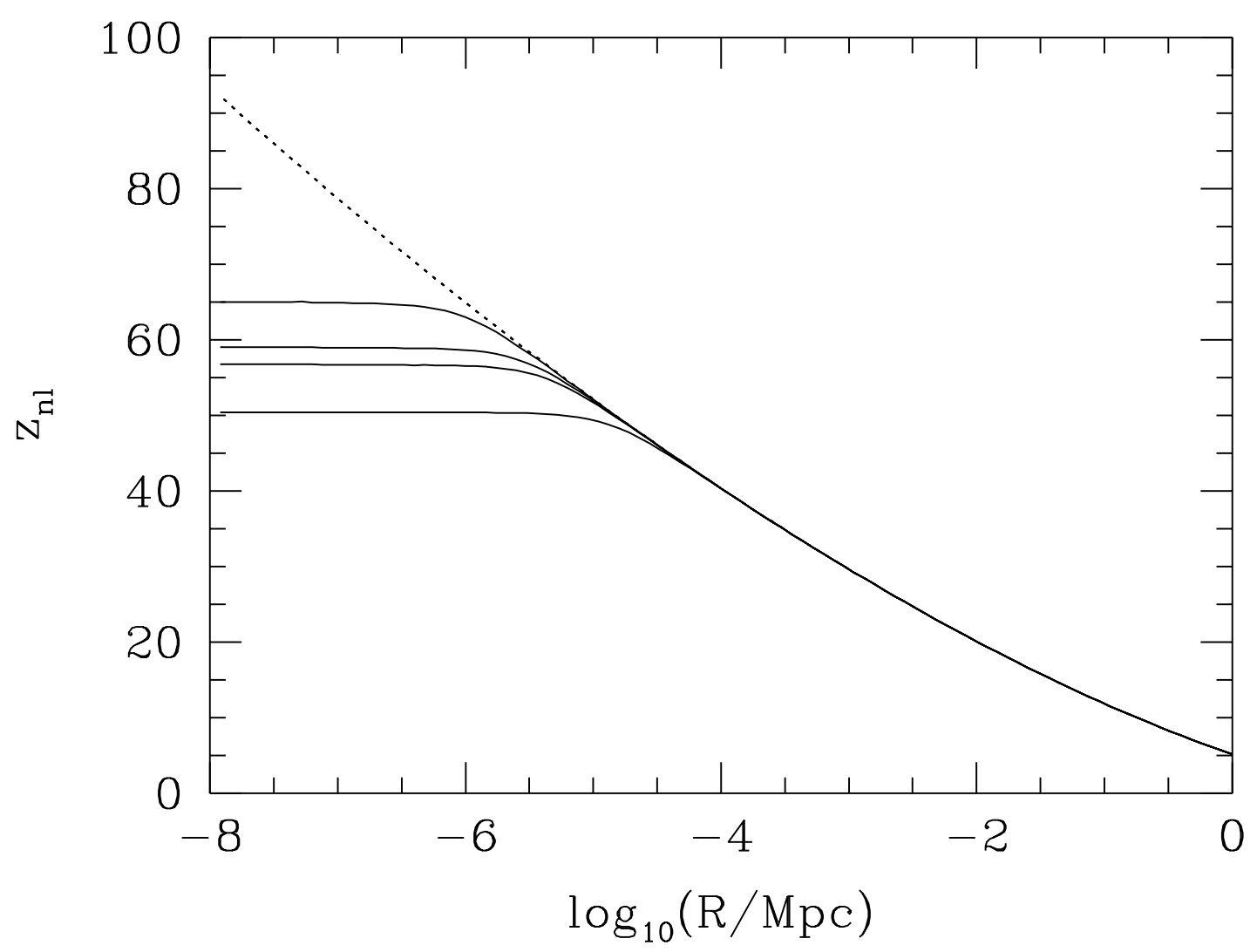

Figure 7. The redshift at which typical fluctuations of comoving scale $R$ become non-linear, $z_{\mathrm{nl}}$, for the WIMP benchmark models discussed in the text (from top to bottom: D, C, B, A). The full lines take into account the effects of collisional damping and free streaming, whereas the dashed line shows the behaviour without a cut-off in the power spectrum. The normalisation is fixed by $\sigma_{8}=0.9$.

a range of inflation models which produce scale-dependent power spectra consistent with the WMAP data $z_{\mathrm{nl}}^{\max }$ for typical fluctuations takes values in the range 40 to 80 . For the best fit WMAP matter density and a scale invariant power spectrum $z_{\mathrm{nl}}^{\max }=60 \pm 10$ with the variation being due to the dependence of the free-streaming scale on the WIMP properties.

The redshift of formation of the very first WIMPy halos is very different from the redshift $z_{\mathrm{nl}}^{\max }$ when hierarchical structure formation starts at a typical place in the universe. In order to make quantitative statements about the first WIMPy halos we need to specify the statistical distribution of density fluctuations. Here we assume that they have a normal (gaussian) distribution, which is justified by two physical arguments. Firstly, we are looking at the very first non-linear objects to form, thus there was no non-linear physics before the rare fluctuations that we are going to discuss enter the non-linear regime. This means that, if the primordial fluctuations are gaussian, the fluctuations from which the first non-linear (rare) objects form should also be close to gaussian. On top of that argument, the central limit theorem (in the limit of large 


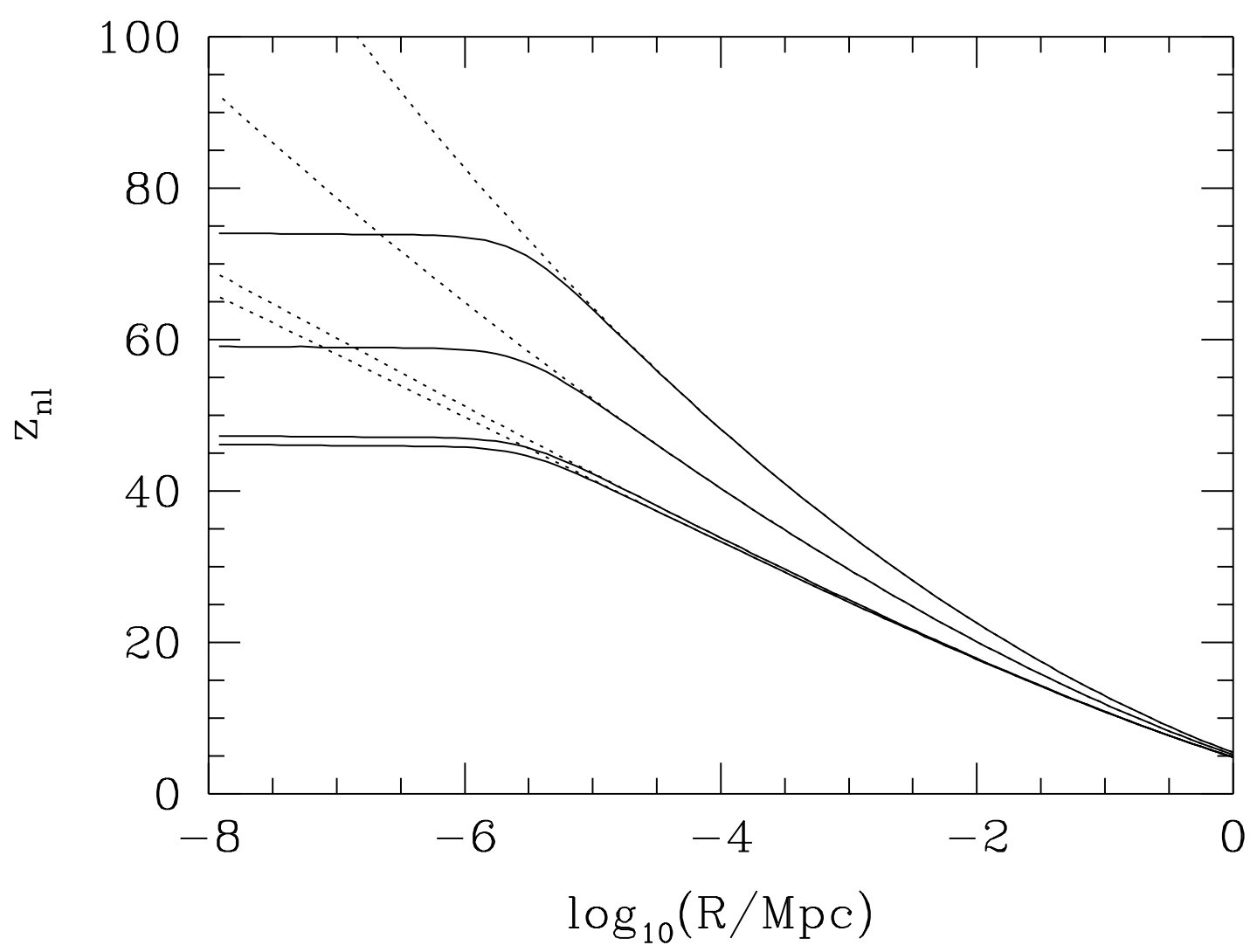

Figure 8. The redshift at which typical fluctuations of comoving scale $R$ become non-linear, $z_{\mathrm{nl}}$, now for the primordial power spectra discussed in the text (from top to bottom: hybrid inflation, scale invariant primordial spectrum, power law and $m^{2} \phi^{2}$ chaotic inflation). The normalisation is fixed by $\sigma_{8}=0.9$.

numbers of distributions - one for each trial volume - the cumulative distribution becomes normal) justifies the use of a gaussian distribution. A typical comoving volume of interest is the mass collection volume of the Milky Way, which is about $1 \mathrm{Mpc}^{3}$. As shown above, the cut-off due to free-streaming implies that the first WIMPy halos have a mass collecting volume of about $1 \mathrm{pc}^{3}$. Thus we are talking about a sample of $\sim 10^{18}$ primary halos.

As a consequence the probability that any one of the $10^{18}$ primary regions within a comoving Milky Way volume has a density that exceeds $N \sigma$ is given by

$$
P\left(\Delta_{\mathrm{m}}(\mathbf{x})>N \sigma\right)=\frac{1}{2}[1-\operatorname{erf}(N / \sqrt{2})] .
$$

Such a primary halo with $\Delta_{\mathrm{m}}=N \sigma$ goes non-linear at

$$
N \sigma\left(R_{\min }, z_{\mathrm{nl}}^{\max }(N)\right)=1 .
$$

As fluctuations grow linear with the scale factor during the matter dominated epoch, this condition provides us with the simple result $z_{\mathrm{nl}}^{\max }(N) \approx N z_{\mathrm{nl}}^{\max }(N=1) \approx(60 \pm 20) N$. Here we consider the uncertainty in the primordial power spectrum and in the WIMP physics. The comoving cut-off scale $R_{\min }$ is independent of the redshift $z_{\mathrm{nl}}$. 
We estimate the size and mass of the first generation of subhalos that form at $z_{\mathrm{nl}}^{\max }$, as well as the size and mass of the very first WIMPy halos, using the spherical collapse model (see e.g. Reference [30]). We should caution that this simplified model has not yet been validated in this regime where the scale dependence of the (processed) power spectrum is relatively weak. The mean CDM mass within a sphere of comoving radius $\mathrm{R}$ is $M(R)=1.6 \times 10^{-7} M_{\odot}\left(\omega_{\mathrm{m}} / 0.14\right)(R / \mathrm{pc})^{3}$. CDM overdensities that go non-linear have mass twice this value i.e. roughly equal to the mass of Mars. These WIMP halos are however much less compact than Mars. The physical size of the first halos at turn-around (when their evolution decouples from the cosmic expansion) is $r=1.05 R /\left[1+z_{\mathrm{nl}}^{\max }(N)\right]$, which is $\sim(0.02 / N)$ pc for $R_{\min }=1$ pc. The first halos then undergo violent relaxation, decreasing in radius by a factor of two so that their present day radius would be of order tens of milli-pc $(N=1)$, comparable to the size of the solar system, and smaller.

A rough estimate of the relevance and chances of survival of the very first WIMPy halos can be made using the present day density contrast of these objects in the spherical collapse model. We find

$$
\Delta=\frac{2 M\left(R_{\min }\right)}{\frac{4 \pi}{3}\left[\frac{r\left(R_{\min }, z_{\mathrm{nl}}^{\max }(N)\right)}{2}\right]^{3} \epsilon_{\mathrm{c} 0}}=3.7(60 \pm 20)^{3} N^{3} .
$$

Remarkably, the result is independent of the comoving size $R_{\text {min }}$. As larger halos form later, their density contrast is smaller, as $\Delta \propto z_{\mathrm{nl}}^{3}$. In figure 9] we plot $\Delta(N)$ for $z_{\mathrm{nl}}^{\max }=40,60$ and 80 and compare it to the density contrast in the galactic disc and in the halo in the solar neighborhood, $\Delta_{\text {disc }}=(0.3$ to 1.2$) 10^{6}$ and $\Delta_{\text {halo }}=(0.2$ to 1.3$) 10^{5}$ [46]. For the typical fluctuations $(N=1)$ we find $\Delta=(0.2$ to 1.8$) 10^{6}$, which is of the same order of magnitude as the local density contrast of the disc. This suggests that the typical first WIMPy halos may not survive the highly non-linear processing which occurs during structure formation.

The situation is very different if we look at the rare fluctuations $(N>1)$, which are characterised by the fact that they go nonlinear much earlier and are thus much denser than other close-by structures. As can be seen in figure 9, e.g. $N=3$ overdense regions lead to a range of $\Delta(N=3)=(0.6$ to 4.9$) 10^{7}$, more than an order of magnitude denser than the local disc and more than two orders of magnitude above the local halo density. Statistically it needs $\sim 740$ comoving $\mathrm{pc}^{3}$ volumes to find one $N=3$ fluctuation. The Milky Way has a (comoving) mass collecting radius of $\sim 1 \mathrm{Mpc}$ therefore, if these very first WIMP halos survive, there would be roughly $10^{15}\left(10^{11}\right)$ 'rare $N=3(6)$ ' WIMPy subhalos within the Milky Way. Assuming the Milky Way has a volume of the order of $(100 \mathrm{kpc})^{3}$, there would be, on average, one 'rare' $N=3(6)$ subhalo in each $\mathrm{pc}^{3}[(20$ $\mathrm{pc})^{3}$ ] volume.

What are the possible implications for direct and indirect dark matter searches? As we discussed above, the comparison of the local halo and disc density contrasts with those expected for the first typical fluctuations indicates that most of the first generation of halos will likely be destroyed during the structure formation process. In this case, apart from within the few rare surviving subhalos, the direct detection event rate will 


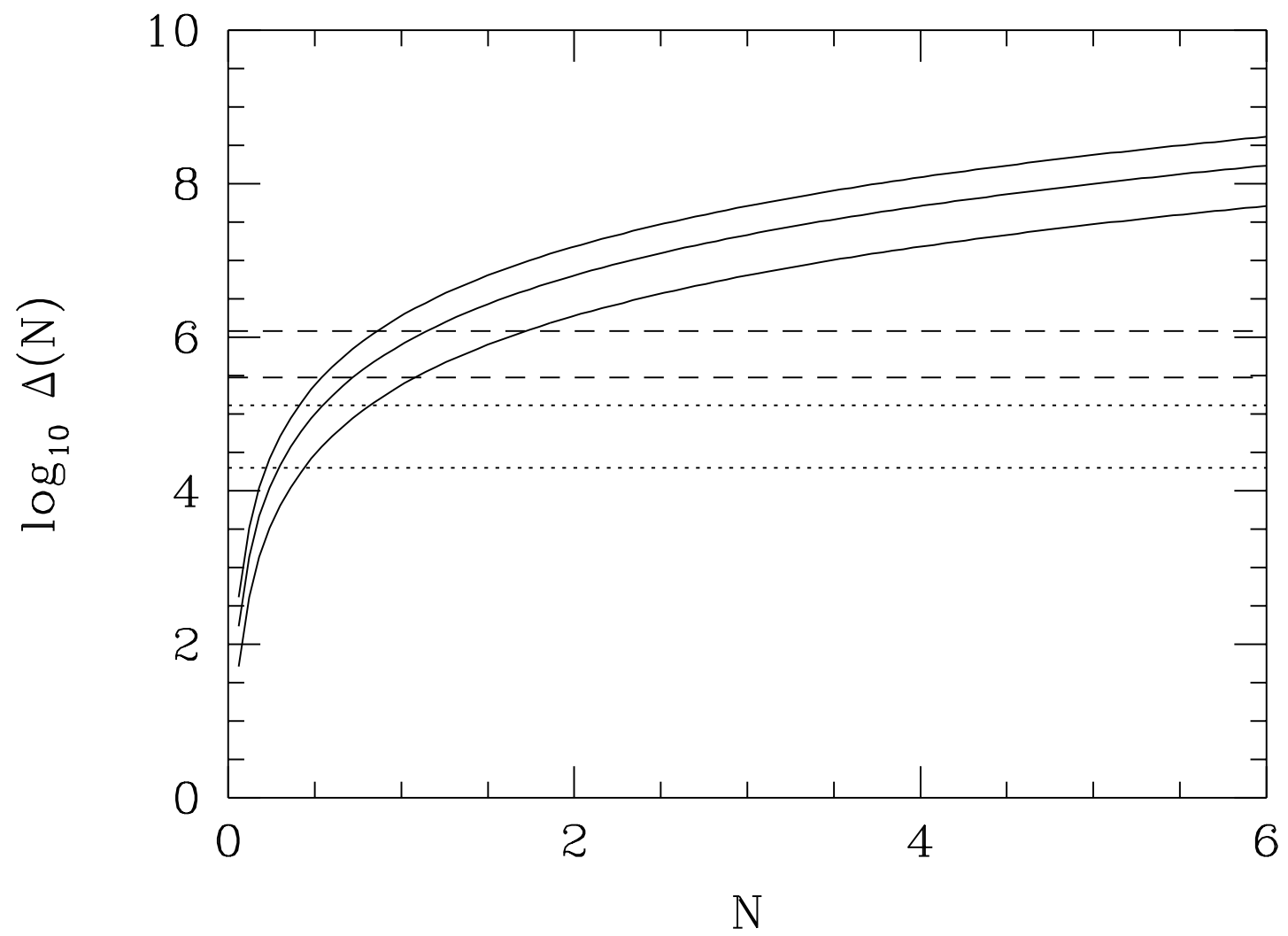

Figure 9. The present day (mean) density contrast of $N \sigma$ fluctuations $\Delta(N)$ for, from top to bottom, $z_{\mathrm{nl}}^{\max }(N=1)=80,60,40$. The dashed (dotted) lines indicate the range of values for the density contrast of the Milky Way disc (halo) in the solar neighbourhood. The normalisation is fixed by $\sigma_{8}=0.9$.

be only slightly lower than found using the standard approach, which assumes a smooth dark matter distribution. The consequences for indirect detection could be much more dramatic. As the indirect detection rate scales with the square of the density contrast, the 'rare' subhalos discussed above could provide 'bright' point sources in the solar neighborhood, which eventually could dominate other sources (e.g. the center of the Milky Way). A detailed investigation of the effect on the expected direct and indirect dark matter search rates, as well as a detailed investigation of the survival probability of rare fluctuations, is beyond the scope of this paper.

\section{Discussion}

Extensive experimental efforts are being devoted to detecting WIMPs, either directly in the lab or indirectly via their annihilation products. Results from these searches are usually quoted in terms of limits on the relevant WIMP interaction cross section. The expected event rates in fact depend, in some cases very sensitively, on the dark matter distribution and hence the formation history of the Milky Way. 
Collisional damping and free-streaming produce a cut-off in the power spectrum at a co-moving scale around $1 \mathrm{pc}$ and set the scale of the first, smallest WIMP halos to from. In this paper we have calculated the damping processes for generic WIMPs and examined the effect of scale dependence of the primordial perturbation spectrum. We have fixed the parameters of the $\Lambda$ CDM model, $\omega_{\mathrm{m}}, \omega_{\mathrm{b}}, \Omega_{\Lambda}$ and $A$ (resp. $\sigma_{8}$ ), to the best-fit WMAP values. A discussion of the effect of varying CDM density has been provided in our previous work [14].

We have found that the smallest scale fluctuations go non-linear (more precisely typical one-sigma fluctuations collapse to from dark matter halos) at a red-shift in the range 40-80, with the first WIMP halos forming significantly earlier from rare large fluctuations. Finally we estimated the properties of both the typical and first small halos to form using the spherical collapse model. The mass of the halos is independent of the size of the fluctuation from which they form, however the first rare fluctuations to collapse form more compact, denser halos.

Neglecting collisional damping and free streaming of WIMPs would result in monotonically increasing power of density fluctuations on small scales. As a consequence, there would be a divergence of the energy density of the fluctuations at small scales and some kind of regularization procedure would be required to make the hierarchical picture of structure formation well-defined. The collisional damping and free streaming of WIMPs regularize the power spectrum by providing a physical cut-off scale.

Numerical studies of the formation of the first WIMPy halos have recently been carried out by Diemand et al. [16]. The subsequent evolution of these halos, and the resulting present day dark matter distribution remains an important outstanding issue.

\section{Acknowledgments}

We would like to thank Lars Bergström, Joakim Edsjö, Ariel Goobar, Abraham Loeb, Ben Moore, Kerstin Paech, Mia Schelke, James Taylor, Tom Theuns, Licia Verde and Matias Zaldarriaga for valuable discussions. AMG was supported by the Swedish Research Council and PPARC. SH was supported by the Wenner-Gren Foundation.

\section{References}

[1] Spergel D N et al First year Wilkinson Microwave Anisotropy Probe (WMAP) observations: determination of cosmological parameters, 2003, Astrophys. J. Suppl. 148175 astro-ph/0302209

[2] Tytler D et al Review of big bang nucleosynthesis and primordial abundances, 2000, Phys. Scr. T 8512 astro-ph/0001318

[3] Percival W J et al The 2dF galaxy redshift survey: The power spectrum and the matter content of the universe, 2001, Mon. Not. Roy. Astron. Soc. 3271297 astro-ph/0105252; The SDSS Collaboration: Tegmark M et al The 3-d power spectrum of galaxies from the SDSS, 2004, Astrophys. J. 606702 astro-ph/0310723

[4] Gondolo P, Introduction to non-baryonic dark matter, 2004, astro-ph/0403064

[5] Dimopoulos S, LHC, SSC and the Universe, 1990, Phys. Lett. B 246347 
[6] Drukier A K, Freese K and Spergel D N, Detecting cold dark matter candidates, 1986, Phys. Rev. D 333495

[7] Bergström L, Non-baryonic dark matter-observational evidence and detection methods, 2000, Rept. Prog. Phys. 63793 [astro-ph/002126]

[8] Silk J and Stebbins A, Clumpy cold dark matter, 1993, Astrophys. J 411439

[9] Moore B et al Dark matter in Draco and the Local Group: implications for direct detection experiments, 2001, Phys. Rev. D 64063508 astro-ph/0106271; Green A M, Effect of halo modelling on WIMP exclusion limits, 2002, Phys. Rev. D 66083003 astro-ph/0304446; Green A $\mathrm{M}$, Effect of realistic astrophysical input on the phase and shape of the WIMP annual modulation signal, 2003, Phys. Rev. D 68023004 erratum-ibid 69, 109902 astro-ph/0207366

[10] Bergström L, Edsjö J, Gondolo P. and Ullio P, Clumpy neutralino dark matter, 1999, Phys. Rev. D 59043506 astro-ph/9806072; Bergström L, Edsjö J. and Gunnarsson C, Neutralino gammaray signals from accreting halo dark matter, 2001, Phys. Rev. D 63083515 astro-ph/0012346; Calcaneo-Roldan C and Moore B, The surface brightness of dark matter: unique signatures of neutralino annihilation, 2000, Phys. Rev. D 62123005 astro-ph/0010056

[11] Ullio P, Bergström L, Edsjö J and Lacey C, Cosmological dark matter annihilations into gammarays - a closer look, 2002, Phys. Rev. D 66123502 astro-ph/0207125; Taylor J E and Silk J, The clumpiness of cold dark matter: implications for the annihilation signal, 2003, Mon. Not. Roy. Astron. Soc. 339505

[12] Hofmann S, Schwarz D J and Stöcker H, Damping scales of neutralino cold dark matter, 2001, Phys. Rev. D 64083507 astro-ph/0104173

[13] Schwarz D J, Hofmann S and Stöcker H, Damping of inhomogeneities in neutralino dark matter, 2001, PrHEP hep2001 204 astro-ph/0110601

[14] Green A M, Hofmann S and Schwarz D J, The power spectrum of SUSY-CDM on sub-galactic scales, 2004, Mon. Not. Roy. Astron. Soc. 353, L23 astro-ph/0309621

[15] Berezinsky V, Dokuchaev V and Eroshenko Y, Small-scale clumps in the galactic halo and dark matter annihilation, 2003, Phys. Rev. D 68103003 astro-ph/0301551

[16] Diemand J, Moore B and Stadel J, Earth-mass dark-matter haloes as the first structures in the early Universe, 2005, Nature 433, 389 astro-ph/0501589

[17] Zhao H, Taylor J E, Silk J and Hooper D, Earth-mass dark halos are torn into dark mini-streams by stars, 2005, astro-ph/0502049; Moore B, Diemand J, Stadel J and Quinn T, On the survival and disruption of Earth mass CDM micro-halos, 2005, astro-ph/0502213

[18] Loeb A and Zaldarriaga M, The small-scale power spectrum of cold dark matter, 2005, Phys. Rev. D 71103520 astro-ph/0504112

[19] Jungman G, Kamionkowski M and Griest K, Supersymmetric dark matter, 1996, Phys. Rep. 267 195

[20] Schmid C, Schwarz D J and Widerin P, Amplification of cosmological inhomogeneities by the QCD transition, 1999, Phys. Rev. D 59043517 astro-ph/9807257

[21] Mukhanov V F, Feldman H A and Brandenberger R, Theory of cosmological perturbations, 1992, Phys. Rep. 215203

[22] Bardeen J M, Gauge-invariant cosmological perturbations, 1980, Phys. Rev. D 22 1882; Cosmological perturbations, from quantum fluctuations to large scale structure, 1989, in Particle Physics and Cosmology, eds. Fang, L \& Zee, A (Gordon and Breach, New York)

[23] Martin J and Schwarz D J, The influence of cosmological transitions on the evolution of density perturbations, 1998, Phys. Rev. D 573302 gr-qc/9704049; Wands D, Malik K A, Lyth D H and Liddle A R, A new approach to the evolution of cosmological perturbations on large scales, 2000, Phys. Rev. D 62043527 astro-ph/0003278

[24] Abramowitz M and Stegun I A, Handbook of mathematical functions, 1970 (Dover Publications Inc., New York)

[25] Bernstein J, Kinetic theory in the expanding universe, 1988 (Cambridge University Press, Cambridge and New York) 
[26] Gradshteyn I S, Ryzhik I M Table of Integrals, Series, and Products, 1965 (Academic Press, New York) p. 495

[27] $\mathrm{Hu} \mathrm{W}$ et al The effect of physical assumptions on the calculation of microwave background anisotropies, 1995, Phys. Rev. D 525498 astro-ph/9505043

[28] Yamamoto K, Sugiyama N and Sato H, Cosmological baryon sound wave coupled with the primeval radiation, 1997, Phys. Rev. D 567566 astro-ph/9709209

[29] Yamamoto K, Sugiyama N and Sato H, Evolution of small scale cosmological baryon perturbations and matter transfer functions, 1998, Astrophys. J. 501442 astro-ph/9709247

[30] Padmanabhan T, Structure formation in the universe, 1993 (Cambridge University Press, Cambridge)

[31] Weinberg S, Cosmological fluctuations of small wavelength, 2002, Astrophys. J $\mathbf{5 8 1} 810$ astro-ph/0207375

[32] $\mathrm{Hu} \mathrm{W}$ and Sugiyama N, Small-scale perturbations: an analytic approach, 1996, Astrophys. J. 471 542 astro-ph/9510117

[33] Peebles P J E and Yu J T, Primeval adiabatic perturbation in an expanding universe, 1970, Astrophys. J. 162 815; Zakharov A V, Effect of collisionless particles on the growth of gravitational perturbations in an isotropic universe, 1979, Zh. Eksp. Teor. Fiz. 77434 [Sov. Phys. JETP 50, 221]

[34] Rebhan A K and Schwarz D J, Kinetic versus thermal-field theory approach to cosmological perturbations, 1994 Phys. Rev. D 502541

[35] Bertschinger E, http://arctus.mit.edu/cosmics/

[36] Carroll S M, Press W H and Turner E L, The cosmological constant, 1991, Ann. Rev. Astron. and Astrophys. 30499

[37] Erdélyi A, Higher transcendental functions, vol I, 1953 (McGraw-Hill Book Company, New York)

[38] Eisenstein D J, An analytic expression for the growth function in a flat universe with cosmological constant, 1997, astro-ph/9709054

[39] Verde L et al First year Wilkinson Microwave Anisotropy Probe (WMAP) observations: parameter estimation methodology, 2003 Astrophys. J. Suppl 148195 astro-ph/0302218

[40] Schwarz D J and Terrero-Escalante C A, Primordial fluctuations and cosmological inflation after WMAP 1.0, 2004, JCAP 0408, 003 hep-ph/0403129

[41] Schwarz D J, Terrero-Escalante C A and Garcia A A, Higher order corrections to primordial spectra from cosmological inflation, 2001, Phys. Lett. B517, 243 astro-ph/0106020

[42] Leach S M, Liddle A R, Martin J and Schwarz D J, Cosmological parameter estimation and the inflationary cosmology, 2002, Phys. Rev. D 66023515 astro-ph/0202095

[43] Stewart E D and Gong J, The density perturbation power spectrum to second order in the slow roll expansion, 2001, Phys. Lett. B510, 1 astro-ph/0101225

[44] Leach S M and Liddle A R, Constraining slow-roll inflation with WMAP and 2dF, 2003, Phys. Rev. D 68, 123508 astro-ph/0306305

[45] Leach S M and Liddle A R, How long before the end of inflation were observable perturbations produced?, 2003, Phys. Rev. D 68, 103503 astro-ph/0305263

[46] Eidelman S et al. Review of Particle Physics, 2004, Phys. Lett. B592, 1 http://pdg.lbl.gov/2004/reviews/contents_sports.html 